\title{
EXISTENCE OF STRONG MILD SOLUTION OF THE NAVIER-STOKES EQUATIONS IN THE HALF SPACE WITH NONDECAYING INITIAL DATA
}

\author{
Hyeong-OhK Bae And Bum Ja Jin
}

\begin{abstract}
We construct a mild solutions of the Navier-Stokes equations in half spaces for nondecaying initial velocities. We also obtain the uniform bound of the velocity field and its derivatives.
\end{abstract}

\section{Introduction}

Consider the Navier-Stokes equations in the $n$-dimensional half space $\mathbb{R}_{+}^{n}$, $n \geq 2$;

$$
\begin{cases}\frac{\partial}{\partial t} u-\Delta u+\nabla p=-\operatorname{div}(u \otimes u) & \text { for } x \in \mathbb{R}_{+}^{n}, t>0, \\ \nabla \cdot u=0 & \text { for } x \in \mathbb{R}_{+}^{n}, t>0, \\ \left.u\right|_{t=0}=a & \text { for } x \in \mathbb{R}_{+}^{n}, \\ \left.u\right|_{x_{n}=0}=0 & \text { for } t>0 .\end{cases}
$$

Here, $u=u(x, t)=\left(u_{1}(x, t), \ldots, u_{n}(x, t)\right)$ and $p=p(x, t)$ represent the unknown velocity vector field and the pressure, respectively, of the fluid, and $a$ is a given initial velocity vector field, with $\nabla \cdot a=0$ and $\left.a\right|_{x_{n}=0}=0$, which may not decay at infinity.

In this paper, we concentrate the existence and the uniqueness of solutions for nondecaying initial data $a$.

There are several results in the whole space $\mathbb{R}^{n}$ for such a problem. It is well-known that for $a \in L^{\infty}\left(\mathbb{R}^{n}\right)$ there is a unique local in time solution $u$ for the Navier-Stokes equations with

$$
p=\sum_{i, j=1}^{n} R_{i} R_{j} u_{i} u_{j}
$$

Received August 30, 2010.

2010 Mathematics Subject Classification. 35Q30, 76D05.

Key words and phrases. Navier-Stokes, nondecaying data, existence, uniqueness.

This research was supported by Basic Science Research Program through the National Research Foundation of Korea(NRF) funded by the Ministry of Education, Science and Technology 2009-0072196. 
where $R_{j}=(-\Delta)^{-1 / 2} \frac{\partial}{\partial x_{j}}$ is the Riesz operator. Refer to [1] and [7] for survey and references. In $\mathbb{R}^{2}$, this solution can be extended globally in time [7].

In [10], it is shown that for linearly growing initial data of the form $a=$ $b-M x$, the Navier-Stokes equations admit a unique local in time solution in $L^{p}\left(\mathbb{R}^{n}\right)$, where $M$ is an $n \times n$ matrix with constant real entries and $b \in L^{p}\left(\mathbb{R}^{n}\right)$.

As mentioned in [11], for $u=g(t)$, then $(u, p)$ always solves (1.1) in $\mathbb{R}^{n}$ with $p=-g^{\prime}(t) \cdot x$, no matter what function $g$ is. Therefore, solution $u$ with a constant initial data is not unique without assuming (1.2). In [11], it is shown that for initial data $a \in L^{\infty}\left(\mathbb{R}^{n}\right)$ with $\nabla \cdot a=0$, if $(u, p)$ is a solution with

$$
u \in L^{\infty}\left((0, T) \times \mathbb{R}^{n}\right), \quad p \in L_{\mathrm{loc}}^{1}([0, T) ; \mathrm{BMO}),
$$

then $(u, \nabla p)$ is uniquely determined by $a$, and $\nabla p=\sum \nabla R_{i} R_{j} u_{i} u_{j}$ in the distributional sense. Recently, in [14], the BMO space for the pressure is replaced by the generalized Campanato space.

Up to now, in the half space case, the local in time existence of mild solution of the Navier-Stokes problems is provided in [16] with continuous, bounded initial data. In this article, we also work on the existence and the uniqueness of (strong) mild solutions for the Navier-Stokes equations with nondecaying initial data in $\mathbb{R}_{+}^{n}$. See also [9] for the rotating Navier-Stokes equations with nondecaying data.

Recently, we have an information of P. Maremonti [12]'s existence result on the Navier-Stokes equations with bounded and continuous initial data. However, our result is different in the sense that our initial data is assumed to be just bounded, and our estimate of the linear and nonlinear operator is from different technique.

We transform (1.1) into the abstract differential equations

$$
\frac{\partial}{\partial t} u+A u=-\mathbb{P} \operatorname{div}(u \otimes u),
$$

where $A=-\mathbb{P} \Delta$ is the Stokes operator and $\mathbb{P}$ is the Leray projection operator, which is represented as

$$
\mathbb{P} f(x) \equiv f(x)+\nabla_{x} \int_{\mathbb{R}_{+}^{n}} \nabla_{y} \mathcal{G}(x, y) \cdot f(y) d y,
$$

when $\left.f_{n}\right|_{x_{n}=0}=0$. Here,

$$
\mathcal{G}(x, y) \equiv N(x-y)+N\left(x-y^{*}\right)
$$

where $y^{*} \equiv\left(y_{1}, \ldots, y_{n-1},-y_{n}\right), N(x) \equiv \frac{1}{n(2-n) \omega_{n}}|x|^{2-n}$, if $n \geq 3$, and $N(x) \equiv$ $\frac{1}{2 \pi} \ln |x|$ if $n=2$, and $\omega_{n}$ denotes the surface area of the unit sphere in $\mathbb{R}^{n}$.

Using the solution operator of the Stokes equations in $\mathbb{R}_{+}^{n}$, the solution of (1.3) is formally expressed in the integral form

$$
u(t)=e^{-A t} a-\int_{0}^{t} e^{-A(t-s)} \mathbb{P} \operatorname{div}(u \otimes u)(s) d s .
$$


We define a bilinear operator $B$ by

$$
B(u, v)(t) \equiv-\int_{0}^{t} e^{-A(t-s)} \mathbb{P} \operatorname{div}(u \otimes v)(s) d s .
$$

A mild solution of the Navier-Stokes equations is defined as following.

Definition 1.1. Let $a \in S^{\prime}$. A measurable function $u \in L_{\mathrm{loc}}^{\infty}\left((0, T) \times \mathbb{R}_{+}^{n}\right)$ is called a mild solution of Navier-Stokes equations (1.1) on $(0, T)$, if $u$ satisfies

$$
u(t)=e^{-t A} a+B(u, u)(t) \text { on }(0, T)
$$

in the sense of distribution. Here, $S^{\prime}$ is the set of distributions.

Solonnikov [16] has expressed the solution operator of the Stokes equations in $\mathbb{R}_{+}^{n}$ in the integral form

$$
e^{-A t} a \equiv \int_{\mathbb{R}_{+}^{n}} G(x, y, t) \cdot a(y) d y,
$$

where $G=\left(G_{i j}\right)_{i, j=1, \ldots, n}$ is defined by

$$
\begin{aligned}
G_{i j}(x, y, t) \equiv & \delta_{i j}\left(\Gamma(x-y, t)-\Gamma\left(x-y^{*}, t\right)\right) \\
& +4\left(1-\delta_{j n}\right) \frac{\partial}{\partial x_{j}} \int_{0}^{x_{n}} \int_{\mathbb{R}^{n-1}} \frac{\partial}{\partial x_{i}} N(x-z) \Gamma\left(z-y^{*}, t\right) d z .
\end{aligned}
$$

The function $\Gamma(x, t)$ is the $n$ dimensional Gaussian kernel defined by $\Gamma(x, t) \equiv$ $\Gamma_{t}(x) \equiv \frac{1}{(2 \pi t)^{n / 2}} e^{-\frac{|x|^{2}}{4 t}}$.

Stokes solutions in $\mathbb{R}_{+}^{n}$ have also been derived in [17]. This solution formula have been used in the $L^{q}$ framework, mainly for $1<q<\infty$ (see [4, 6], see also $[2,3,8,15]$ for the $L^{1}$ or $L^{\infty}$ estimates of the Stokes flow or its gradient). The solution formula in [16] has been used mainly for $L^{\infty}$ framework (see $[5,13,16]$ ).

The goal of this paper is to construct strong mild solution of the NavierStokes equations in $\mathbb{R}_{+}^{n}$ when the initial data is in $L^{\infty}\left(\mathbb{R}_{+}^{n}\right)$. For the construction of the solutions, the following estimate plays important role: for $\mathcal{F}$ with 0 on $x_{n}=0$,

$$
\begin{aligned}
&\left\|e^{-A t} \mathcal{F}\right\|_{L^{\infty}\left(\mathbb{R}_{+}^{n}\right)} \leq C\|\mathcal{F}\|_{L^{\infty}\left(\mathbb{R}_{+}^{n}\right)}, \\
&\left\|\nabla e^{-A t} \mathcal{F}\right\|_{L^{\infty}\left(\mathbb{R}_{+}^{n}\right)} \leq C t^{-\frac{1}{2}}\|\mathcal{F}\|_{L^{\infty}\left(\mathbb{R}_{+}^{n}\right)}, \\
&\left\|e^{-A t} \mathbb{P} \operatorname{div} \mathcal{F}\right\|_{L^{\infty}\left(\mathbb{R}_{+}^{n}\right)} \leq C t^{-\frac{1}{2}}\|\mathcal{F}\|_{L^{\infty}\left(\mathbb{R}_{+}^{n}\right)}, \\
&\left\|\nabla e^{-A t} \mathbb{P} \operatorname{div} \mathcal{F}\right\|_{L^{\infty}\left(\mathbb{R}_{+}^{n}\right)} \leq C t^{-\frac{1}{2}}\|\nabla \mathcal{F}\|_{L^{\infty}\left(\mathbb{R}_{+}^{n}\right)} .
\end{aligned}
$$

Especially, the estimate (1.10) is used for the gradient of the velocity.

The followings are the main theorems.

Theorem 1.2. Let $a \in L^{\infty}\left(\mathbb{R}_{+}^{n}\right)$ be the given vector field diva $=0$ and $\left.a\right|_{x_{n}=0}=$ 0 . Then there is a positive time $T>0$, and there is a mild solution $u \in$ 
$L^{\infty}\left((0, T) \times \mathbb{R}_{+}^{n}\right)$ which satisfies (1.5). Moreover, it holds that for $0<t<T$,

$$
\|u(t)\|_{L^{\infty}\left(\mathbb{R}_{+}^{n}\right)}+t^{\frac{1}{2}}\|\nabla u(t)\|_{L^{\infty}\left(\mathbb{R}_{+}^{n}\right)} \leq C\|a\|_{L^{\infty}\left(\mathbb{R}_{+}^{n}\right)} .
$$

Theorem 1.3. Suppose that $u, v \in L_{\mathrm{loc}}^{\infty}\left((0, T) \times \mathbb{R}_{+}^{n}\right)$ are mild solutions of the Navier-Stokes equations (1.5) with the same initial data. If both $u$ and $v$ satisfy the following conditions

$$
\sup _{0<t<T} t^{\frac{\alpha}{2}}\|u(t)\|_{L^{\infty}}<\infty \text { and } \sup _{0<t<T} t^{\frac{\alpha}{2}}\|v(t)\|_{L^{\infty}}<\infty \text { for some } 0 \leq \alpha<1,
$$

then $u \equiv v$ on $(0, T) \times \mathbb{R}_{+}^{n}$.

We compare our results with those in [16] with several points of view. In [16], to get the short time existence of mild solution, the estimates (1.7)-(1.9) are provided, but (1.10) is not.

In this paper as well as the boundedness of the velocity, we obtain a higher regularity, and hence our solution is not only mild solution but also strong solution for a short time. For the estimate of the gradient of the velocity, it is necessary to have the pointwise estimate of the kernels of the operator $\nabla e^{-t A} \mathbb{P}$ div. Unfortunately, $\nabla$ and $e^{-A t} \mathbb{P}$ are not commutative, while $\nabla_{\bar{x}}$ and $e^{-A t} \mathbb{P}$ are commutative. To obtain (1.10), we first treat the case $\nabla_{\bar{x}} e^{-A t} \mathbb{P} \operatorname{div} \mathcal{F}$, then $\frac{\partial}{\partial x_{n}}\left(e^{-A t} \mathbb{P} \operatorname{div} \mathcal{F}\right)_{n}$, and finally $\frac{\partial}{\partial x_{n}} \overline{e^{-A t} \mathbb{P} \operatorname{div} \mathcal{F}}$, separately. Here $\nabla_{\bar{x}}=$ $\left(\frac{\partial}{\partial x_{1}}, \ldots, \frac{\partial}{\partial x_{n-1}}\right), \bar{f}=\left(f_{1}, \ldots, f_{n-1}\right)$.

We also compare our result with those in [12], where the classical solution $u$ is constructed when the initial velocity $a$ is bounded and continuous and the pointwise estimate of pressure function is also given. For the pointwise estimate of the pressure, the continuity assumption of the given initial data has been necessary.

Acknowledgement. We want to express our thanks to TongKeun Chang for valuable discussions, especially for the estimate of the Stokes operator and bilinear operators.

\section{Preliminaries}

Let us consider the Stokes problems in $\mathbb{R}_{+}^{n}, n \geq 2$ :

$$
\begin{cases}\operatorname{div} u=0 & \text { for } x \in \mathbb{R}_{+}^{n}, t>0 \\ u_{t}-\Delta u+\nabla p=\operatorname{div} \mathcal{F} & \text { for } x \in \mathbb{R}_{+}^{n}, t>0 \\ \left.u\right|_{t=0}=a & \text { for } x \in \mathbb{R}_{+}^{n}, \\ \left.u\right|_{x_{n}=0}=0 & \text { for } t>0\end{cases}
$$

In [16] solution $u$ of the Stokes problems (2.11) is formally given by

$$
u(x, t)=\int_{\mathbb{R}_{+}^{n}} G(x, y, t) \cdot a(y) d y+\int_{0}^{t} d \tau \int_{\mathbb{R}_{+}^{n}} G(x, y, t-\tau) \cdot \mathbb{P} \operatorname{div} \mathcal{F}(y, \tau) d y .
$$


For later usage, we use the notations:

$$
\begin{array}{ll}
\Gamma(x, t) \equiv \Gamma_{t}(x) \equiv \bar{\Gamma}_{t}(\bar{x}) \Gamma_{t}^{n}\left(x_{n}\right) \equiv\left((2 \pi t)^{-\frac{n-1}{2}} e^{-\frac{|\bar{x}|^{2}}{4 t}}\right)\left((2 \pi t)^{-\frac{1}{2}} e^{-\frac{\left|x_{n}\right|^{2}}{4 t}}\right), \\
\|\cdot\|_{\infty} \equiv\|\cdot\|_{L^{\infty}\left(\mathbb{R}_{+}^{n}\right)}, \quad \partial_{x_{i}} \equiv \frac{\partial}{\partial x_{i}} \text { for short. } \\
\nabla_{\bar{x}} \equiv\left(\partial_{x_{1}}, \ldots, \partial_{x_{n-1}}\right), \quad \bar{x}=\left(x_{1}, \ldots, x_{n-1}\right) .
\end{array}
$$

$1_{\mathbb{R}_{+}^{n}}$ denotes the characteristic function which is 1 on $\mathbb{R}_{+}^{n}$, and otherwise 0 .

$\tau_{a}$ a translation operator defined by $\tau_{a} f(x) \equiv f(a-x)$.

Remark 2.1. Suppose that $\operatorname{div} f=0$ with $\left.f_{n}\right|_{x_{n}=0}=0$. Then direct computation shows that

$$
\operatorname{div} e^{-t A} f=0 \text {. }
$$

Proposition 2.1. If $k=1, \ldots, n-1$, then we have

$$
\frac{\partial}{\partial x_{k}} G_{i j}(x, y, t)=-\frac{\partial}{\partial y_{k}} G_{i j}(x, y, t) \text {. }
$$

And we have

$$
\begin{aligned}
\frac{\partial}{\partial x_{n}} G_{i j}(x, y, t)= & \frac{\partial}{\partial y_{n}} G_{i j}(x, y, t)-2 \delta_{i j} \frac{\partial}{\partial y_{n}} \Gamma(x-y, t) \\
& -4\left(1-\delta_{j n}\right) \frac{\partial}{\partial x_{j}} \int_{\mathbb{R}^{n-1}} \frac{\partial}{\partial x_{i}} N\left(\bar{x}-\bar{z}, x_{n}\right) \Gamma\left(\bar{z}-\bar{y}, y_{n}, t\right) d \bar{z} .
\end{aligned}
$$

Proof. Let us consider the case $k \neq n$. Differentiate $G_{i j}$ in terms of $x_{k}$ variable, then we have

$$
\begin{aligned}
\partial_{x_{k}} G_{i j}(x, y, t)= & \delta_{i j} \partial_{x_{k}}\left[\Gamma_{t}\left(x-y^{*}\right)-\Gamma_{t}\left(x-y^{*}\right)\right] \\
& +4\left(1-\delta_{j n}\right) \partial_{x_{k}} \partial_{x_{j}} \int_{0}^{x_{n}} \int_{\mathbb{R}^{n-1}} \partial_{x_{i}} N(x-z) \Gamma_{t}\left(z-y^{*}\right) d z \\
= & \delta_{i j} \partial_{x_{k}}\left[\Gamma_{t}\left(x-y^{*}\right)-\Gamma_{t}\left(x-y^{*}\right)\right] \\
& +4\left(1-\delta_{j n}\right) \partial_{x_{j}} \int_{0}^{x_{n}} \int_{\mathbb{R}^{n-1}} \partial_{x_{i}} N(x-z) \partial_{z_{k}} \Gamma_{t}\left(z-y^{*}\right) d z .
\end{aligned}
$$

The last term of the right hand side of the above identity comes from the fact that

$$
\partial_{x_{k}} \int_{0}^{x_{n}} \int_{\mathbb{R}^{n-1}} \partial_{x_{i}} N(x-z) g(z) d z=\int_{0}^{x_{n}} \int_{\mathbb{R}^{n-1}} \partial_{x_{i}} N(x-z) \partial_{z_{k}} g(z) d z .
$$

Note that $\partial_{y_{k}} \Gamma_{t}(x-y)=-\partial_{x_{k}} \Gamma_{t}(x-y)$ and $\partial_{y_{k}} \Gamma_{t}\left(x-y^{*}\right)=-\partial_{x_{k}} \Gamma\left(x-y^{*}\right)$ if $k \neq n$. Hence we obtain the identity (2.12).

Now consider the case $k=n$. Notice that

$$
\begin{aligned}
& \partial_{x_{n}} \int_{0}^{x_{n}} \int_{\mathbb{R}^{n-1}} \partial_{x_{i}} N(x-z) g(z) d z \\
= & \int_{0}^{x_{n}} \int_{\mathbb{R}^{n-1}} \partial_{x_{i}} \partial_{x_{n}} N(x-z) g(z) d z
\end{aligned}
$$




$$
+\frac{\delta_{i n}}{2 n} g(x)+\int_{\mathbb{R}^{n-1}} \partial_{x_{i}} N(\bar{x}-\bar{z}, 0) g\left(\bar{z}, x_{n}\right) d \bar{z}
$$

and

$$
\begin{aligned}
& -\int_{0}^{x_{n}} \int_{\mathbb{R}^{n-1}} \partial_{x_{i}} \partial_{x_{n}} N(x-z) g(z) d z \\
= & \int_{0}^{x_{n}} \int_{\mathbb{R}^{n-1}} \partial_{x_{i}} \partial_{z_{n}} N(x-z) g(z) d z \\
= & -\int_{0}^{x_{n}} \int_{\mathbb{R}^{n-1}} \partial_{x_{i}} N(x-z) \partial_{z_{n}} g(z) d z \\
& +\frac{\delta_{i n}}{2 n} g(x)+\int_{\mathbb{R}^{n-1}} \partial_{x_{i}} N(\bar{x}-\bar{z}, 0) g\left(\bar{z}, x_{n}\right) d \bar{z} \\
& -\int_{\mathbb{R}^{n-1}} \partial_{x_{i}} N\left(\bar{x}-\bar{z}, z_{n}\right) g(\bar{z}, 0) d \bar{z} .
\end{aligned}
$$

Hence, we obtain that

$$
\begin{aligned}
& \partial_{x_{n}} \int_{0}^{x_{n}} \int_{\mathbb{R}^{n-1}} \partial_{x_{i}} N(x-z) \Gamma\left(z-y^{*}, t\right) d z \\
= & \int_{0}^{x_{n}} \int_{\mathbb{R}^{n-1}} \partial_{x_{i}} N(x-z) \partial_{z_{n}} \Gamma\left(z-y^{*}, t\right) d z \\
& +\int_{\mathbb{R}^{n-1}} \partial_{x_{i}} N\left(\bar{x}-\bar{z}, x_{n}\right) \Gamma\left(\bar{z}-\bar{y}, y_{n}, t\right) d \bar{z} .
\end{aligned}
$$

Note that $\partial_{y_{n}} \Gamma_{t}(x-y)=-\partial_{x_{n}} \Gamma_{t}(x-y)$ and $\partial_{y_{n}} \Gamma_{t}\left(x-y^{*}\right)=\partial_{x_{n}} \Gamma\left(x-y^{*}\right)$.

Therefore if we differentiate $G_{i j}$ in terms of $x_{n}$ variables, then we have

$$
\begin{aligned}
\partial_{x_{n}} G_{i j}(x, y, t)= & -\delta_{i j} \partial_{y_{n}}\left[\left(\Gamma(x-y, t)+\Gamma\left(x-y^{*}, t\right)\right]\right. \\
& +4\left(1-\delta_{j n}\right) \partial_{y_{n}} \partial_{x_{j}} \int_{0}^{x_{n}} \int_{\mathbb{R}^{n-1}} \partial_{x_{i}} N(x-z) \Gamma\left(z-y^{*}, t\right) d z \\
& +4\left(1-\delta_{j n}\right) \partial_{x_{j}} \int_{\mathbb{R}^{n-1}} \partial_{x_{i}} N\left(\bar{x}-\bar{z}, x_{n}\right) \Gamma\left(\bar{z}-\bar{y}, y_{n}, t\right) d \bar{z} \\
= & \partial_{y_{n}} G_{i j}(x, y, t)-2 \delta_{i j} \partial_{y_{n}} \Gamma(x-y, t) \\
& +4\left(1-\delta_{j n}\right) \partial_{x_{j}} \int_{\mathbb{R}^{n-1}} \partial_{x_{i}} N\left(\bar{x}-\bar{z}, x_{n}\right) \Gamma\left(\bar{z}-\bar{y}, y_{n}, t\right) d \bar{z} .
\end{aligned}
$$

Proposition 2.2. Let $x \in \mathbb{R}_{+}^{n}$. Let $f$ be a Hölder continuous function with the exponent $0<\alpha<1$,

$$
[f]_{\alpha}=\sup _{x, z} \frac{|f(x)-f(z)|}{|x-z|^{\alpha}}<\infty .
$$

Then, for $i, j \neq n$ or $i, j=n$, we have

$$
\frac{\partial}{\partial x_{j}} \int_{0}^{x_{n}} \int_{\mathbb{R}^{n-1}} \frac{\partial}{\partial x_{i}} N(x-z) f(z) d z
$$




$$
\begin{aligned}
=- & \frac{\delta_{i j}}{2 n} f(x)+\int_{0}^{x_{n}} \int_{\mathbb{R}^{n-1}} \frac{\partial^{2}}{\partial x_{i} \partial x_{j}} N(x-z) f(z) d z \\
& +\delta_{j n} \int_{\mathbb{R}^{n-1}} \frac{\partial}{\partial x_{i}} N(\bar{x}-\bar{z}) f\left(\bar{z}, x_{n}\right) d \bar{z} .
\end{aligned}
$$

For $i=n, j \neq n$ or $j=n, i \neq n$, we have

$$
\frac{\partial}{\partial x_{j}} \int_{0}^{x_{n}} \int_{\mathbb{R}^{n-1}} \frac{\partial}{\partial x_{i}} N(x-z) f(z) d z=\int_{0}^{x_{n}} \int_{\mathbb{R}^{n-1}} \frac{\partial^{2}}{\partial x_{i} \partial x_{j}} N(x-z) f(z) d z .
$$

Proof. The proof is given in Appendix A.

Now we define the Hardy space $\mathcal{H}^{1}$. Define $\mathcal{N} g(x) \equiv \sup _{s>0}\left|g * \Gamma_{s}(x)\right|$. Let $\mathcal{H}^{1}\left(\mathbb{R}^{n}\right)$ be the space of functions $g$ so that $\mathcal{N} g \in L^{1}\left(\mathbb{R}^{n}\right)$ with the norm $\|g\|_{\mathcal{H}^{1}\left(\mathbb{R}^{n}\right)} \equiv\|\mathcal{N} g\|_{L^{1}\left(\mathbb{R}^{n}\right)}$. The dual space of $\mathcal{H}^{1}\left(\mathbb{R}^{n}\right)$ is defined by $\operatorname{BMO}\left(\mathbb{R}^{n}\right)$. Define $\mathcal{H}^{1}\left(\mathbb{R}_{+}^{n}\right)$ be the space of functions $f$ so that there is $\tilde{f} \in \mathcal{H}^{1}\left(\mathbb{R}^{n}\right)$ with $\left.\tilde{f}\right|_{\mathbb{R}_{+}^{n}}=f$ with the norm $\|f\|_{\mathcal{H}^{1}\left(\mathbb{R}_{+}^{n}\right)} \equiv \inf \left\{\|\tilde{f}\|_{\mathcal{H}^{1}\left(\mathbb{R}^{n}\right)}:\left.\tilde{f}\right|_{\mathbb{R}_{+}^{n}}=f\right\}$, and define $\operatorname{BMO}\left(\mathbb{R}_{+}^{n}\right)$ be the space of functions $f$ so that there is $\tilde{f} \in \operatorname{BMO}\left(\mathbb{R}^{n}\right)$ with $\left.\tilde{f}\right|_{\mathbb{R}_{+}^{n}}=f$ with the norm $\|f\|_{\operatorname{BMO}\left(\mathbb{R}_{+}^{n}\right)}=\inf \left\{\|\tilde{f}\|_{\operatorname{BMO}\left(\mathbb{R}^{\mathrm{n}}\right)}:\left.\quad \tilde{f}\right|_{\mathbb{R}_{+}^{n}}=f\right\}$. It is well known fact that $\nabla \Gamma_{t} \in \mathcal{H}^{1}\left(\mathbb{R}^{n}\right)$. Moreover, in [8] it is shown that $1_{\mathbb{R}_{+}^{n}} \partial_{x_{k}} \Gamma_{t} \in \mathcal{H}^{1}\left(\mathbb{R}^{n}\right)$ for $k \neq n$.

Proposition 2.3. Fix $a \in \mathbb{R}^{n}$. Then $\nabla\left(\tau_{a} \Gamma_{t}\right) \in \mathcal{H}^{1}\left(\mathbb{R}^{n}\right)$ with

$$
\left\|\nabla\left(\tau_{a} \Gamma_{t}\right)\right\|_{\mathcal{H}^{1}} \leq C t^{-\frac{1}{2}}
$$

The above proposition is well known and we omit the proof. Instead, we give the proof that $1_{\mathbb{R}_{+}^{n}} \nabla_{\bar{x}}\left(\tau_{a} \Gamma_{t}\right) \in \mathcal{H}^{1}\left(\mathbb{R}^{n}\right)$.

Lemma 2.4. Fix $a \in \mathbb{R}^{n}$. Then

$$
1_{\mathbb{R}_{+}^{n}} \nabla_{\bar{x}}\left(\tau_{a} \Gamma_{t}\right) \in \mathcal{H}^{1}\left(\mathbb{R}^{n}\right)
$$

with

$$
\left\|1_{\mathbb{R}_{+}^{n}} \nabla_{\bar{x}}\left(\tau_{a} \Gamma_{t}\right)\right\|_{\mathcal{H}^{1}} \leq C t^{-\frac{1}{2}}
$$

Proof. The proof is given in Appendix B, where we follow the idea in [8].

Let $a \in \mathbb{R}_{+}^{n}$. Then $\tau_{a^{*}} \Gamma_{t}(x)=\Gamma_{t}\left(a^{*}-x\right)=\bar{\Gamma}_{t}(\bar{a}-\bar{x}) \Gamma_{t}^{n}\left(a_{n}+x_{n}\right)$. We observe that

$$
\begin{aligned}
\int_{-\infty}^{x_{n}} \Gamma_{t}^{n}\left(a_{n}+x_{n}-y_{n}\right) \Gamma_{s}^{n}\left(y_{n}\right) d y_{n} & \leq e^{-\frac{a_{n}^{2}}{4 t}} \int_{-\infty}^{x_{n}} \Gamma_{t}^{n}\left(x_{n}-y_{n}\right) \Gamma_{s}^{n}\left(y_{n}\right) d y_{n} \\
& \leq e^{-\frac{a_{n}^{2}}{4 t}} \int_{-\infty}^{\infty} \Gamma_{t}^{n}\left(x_{n}-y_{n}\right) \Gamma_{s}^{n}\left(y_{n}\right) d y_{n} \\
& =e^{-\frac{a_{n}^{2}}{4 t}} \Gamma_{s+t}^{n}\left(x_{n}\right) .
\end{aligned}
$$

Then, we also have the following estimate. 
Corollary 2.5. Fix $a=\left(a_{1}, \ldots, a_{n}\right) \in \mathbb{R}_{+}^{n}$. Then we have

$$
\left\|1_{\mathbb{R}_{+}^{n}} \nabla_{\bar{x}}\left(\tau_{a^{*}} \Gamma_{t}\right)\right\|_{\mathcal{H}^{1}} \leq C t^{-\frac{1}{2}} e^{-\frac{a_{n}^{2}}{4 t}} .
$$

Lemma 2.6. Fix $a=\left(\bar{a}, a_{n}\right), b=\left(\bar{b}, a_{n}\right) \in \mathbb{R}_{+}^{n}$. Let $0<\alpha<1$. Then we have

$$
\left\|1_{\mathbb{R}_{+}^{n}}\left[\tau_{a^{*}} \Gamma_{t}-\tau_{b^{*}} \Gamma_{t}\right]\right\|_{\mathcal{H}^{1}} \leq C t^{-\alpha / 2} e^{-\frac{a_{n}^{2}}{4 t}}|\bar{a}-\bar{b}|^{\alpha} .
$$

Proof. The proof is given in Appendix C.

Remark 2.2. In Lemma 2.4, Corollary 2.5, and Lemma 2.6, the results hold true even if we replace $1_{\mathbb{R}_{+}^{n}}$ by $\mathbb{R}^{n-1} \times K=\left\{x_{n} \in K\right\}$ for any one dimensional open set $K \subset \mathbb{R}$. Moreover, making use of the properties of the Gaussian kernels, we have

and

$$
\begin{gathered}
\left\|1_{\left\{x_{n} \in K\right\}} \nabla_{x}^{k} \nabla_{\bar{x}}\left(\tau_{a} \Gamma_{t}\right)\right\|_{\mathcal{H}^{1}} \leq C t^{-\frac{k+1}{2}} \\
\left\|1_{\left\{x_{n} \in K\right\}} \nabla_{x}^{k} \nabla_{\bar{x}}\left(\tau_{a^{*}} \Gamma_{t}\right)\right\|_{\mathcal{H}^{1}} \leq C t^{-\frac{k+1}{2}} e^{-\frac{a_{n}^{2}}{4 t}}
\end{gathered}
$$

$$
\left\|1_{\left\{x_{n} \in K\right\}} \nabla_{x}^{k}\left[\tau_{a^{*}} \Gamma_{t}-\tau_{b^{*}} \Gamma_{t}\right]\right\|_{\mathcal{H}^{1}} \leq C t^{-\frac{k+\alpha}{2}} e^{-\frac{a_{n}^{2}}{4 t}}|\bar{a}-\bar{b}|^{\alpha} .
$$

Lemma 2.7. Let $j=1, \ldots, n-1$ and $i=1, \ldots, n$. Then we have

$$
\begin{aligned}
& \int_{\mathbb{R}_{+}^{n}}\left|\int_{0}^{x_{n}} \int_{\mathbb{R}^{n-1}} \frac{\partial^{2}}{\partial z_{i} \partial z_{j}} N(x-z) f(z, y) d z\right| d y \\
\leq & C \sup _{x \in \mathbb{R}_{+}^{n}} \int_{\mathbb{R}_{+}^{n}}|f(x, y)| d y \\
& +C x_{n}^{\alpha} \sup _{x_{n} / 2<z_{n}<x_{n}} \sup _{\bar{z} \in \mathbb{R}^{n-1}} \frac{\int_{\mathbb{R}_{+}^{n}}\left|f\left(\bar{z}, z_{n}, y\right)-f\left(\bar{x}, z_{n}, y\right)\right| d y}{|\bar{x}-\bar{z}|^{\alpha}} .
\end{aligned}
$$

Proof. The proof is given in Appendix D.

\section{Estimate of $\nabla e^{-t A} \mathbb{P} \nabla \cdot \mathcal{F}$.}

The following is well known (see Solonnikov [16] and Shimizu [15]).

\section{Proposition 3.1.}

$$
\begin{aligned}
\left\|\nabla^{k} e^{-A t} f\right\|_{L^{\infty}\left(\mathbb{R}_{+}^{n}\right)} & \leq C t^{-\frac{k}{2}}\|f\|_{L^{\infty}\left(\mathbb{R}_{+}^{n}\right)}, \\
\left\|e^{-A t} \mathbb{P} \operatorname{div} \mathcal{F}\right\|_{L^{\infty}\left(\mathbb{R}_{+}^{n}\right)} & \leq C t^{-\frac{1}{2}}\|\mathcal{F}\|_{L^{\infty}\left(\mathbb{R}_{+}^{n}\right)} .
\end{aligned}
$$

In this section we would like to show that

$$
\left\|\nabla e^{-A t} \mathbb{P} \operatorname{div} \mathcal{F}\right\|_{\infty} \leq C t^{-\frac{1}{2}}\|\nabla \mathcal{F}\|_{\infty} .
$$

Recall Proposition 2.1 that

$$
\nabla_{\bar{x}} e^{-t A} \mathbb{P} \nabla \cdot \mathcal{F}=e^{-t A} \nabla_{\bar{x}} \mathbb{P} \nabla \cdot \mathcal{F} .
$$

We have the identity

$$
\nabla_{\bar{x}} \mathbb{P} \nabla \cdot \mathcal{F}=\mathbb{P} \nabla \cdot\left[\nabla_{\bar{x}} \mathcal{F}\right],
$$


since for $k \neq n$

$$
\begin{aligned}
\partial_{x_{k}}(\mathbb{P} f)_{j}(x) & =\partial_{x_{k}} f_{j}(x)+\partial_{x_{k}} \partial_{x_{j}} \int_{\mathbb{R}_{+}^{n}} \nabla_{y} \mathcal{G}(x, y) \cdot f(y) d y \\
& =\partial_{x_{k}} f_{j}(x)+\partial_{x_{j}} \int_{\mathbb{R}_{+}^{n}} \nabla_{y} \mathcal{G}(x, y) \cdot \partial_{y_{k}} f(y) d y
\end{aligned}
$$

Applying the known result (3.14) of Proposition 3.1, we have the following estimate.

\section{Lemma 3.2.}

$\left\|\nabla_{\bar{x}} e^{-A t} \mathbb{P} \operatorname{div} \mathcal{F}\right\|_{L^{\infty}\left(\mathbb{R}_{+}^{n}\right)}=\left\|e^{-A t} \mathbb{P} \operatorname{div} \nabla_{\bar{x}} \mathcal{F}\right\|_{L^{\infty}\left(\mathbb{R}_{+}^{n}\right)} \leq c t^{-\frac{1}{2}}\left\|\nabla_{\bar{x}} \mathcal{F}\right\|_{L^{\infty}\left(\mathbb{R}_{+}^{n}\right)}$.

From the divergence free property of $e^{-A t} \mathbb{P} \operatorname{div} \mathcal{F}$, we have that

$$
\partial_{x_{n}}\left(e^{-A t} \mathbb{P} \operatorname{div} \mathcal{F}\right)_{n}=-\sum_{j=1}^{n-1} \partial_{x_{j}}\left(e^{-A t} \mathbb{P} \operatorname{div} \mathcal{F}\right)_{j} .
$$

Hence we have the following estimate.

\section{Lemma 3.3.}

$$
\left\|\partial_{x_{n}}\left(e^{-A t} \mathbb{P} \operatorname{div} \mathcal{F}\right)_{n}\right\|_{L^{\infty}\left(\mathbb{R}_{+}^{n}\right)} \leq c t^{-\frac{1}{2}}\left\|\nabla_{\bar{x}} \mathcal{F}\right\|_{L^{\infty}\left(\mathbb{R}_{+}^{n}\right)} .
$$

Now let us consider the case $i \neq n$. Denote by $H=\left(H_{i j k}\right)_{i, j, k=1}^{n}$ the kernel tensors of the operator $e^{-t A} \mathbb{P}$ div. Then

$$
\left[e^{-t A} \mathbb{P} \operatorname{div} \mathcal{F}\right]_{i}(x)=\int_{\mathbb{R}_{+}^{n}} H_{i j k}(x, y, t) F_{j k}(y) d y .
$$

Direct computation shows that

$$
H_{i j k}(x, y, t)=-\partial_{y_{k}} G_{i j}(x, y, t)+\sum_{l=1}^{n} \partial_{y_{j}} \int_{\mathbb{R}_{+}^{n}} \partial_{y_{k}} \mathcal{G}(z, y) \partial_{z_{l}} G_{i l}(x, z, t) d z .
$$

Moreover, since $G_{i n}=0$ if $i \neq n$, we have

$$
H_{i j k}(x, y, t)=-\partial_{y_{k}} G_{i j}(x, y, t)+\sum_{l=1}^{n-1} \partial_{y_{j}} \int_{\mathbb{R}_{+}^{n}} \partial_{y_{k}} \mathcal{G}(z, y) \partial_{z_{l}} G_{i l}(x, z, t) d z .
$$

From Proposition 2.1, we have

$$
\begin{aligned}
\partial_{x_{n}} G_{i l}(x, z, t)= & \partial_{z_{n}} G_{i l}(x, z, t)-2 \delta_{i l} \partial_{z_{n}} \Gamma(x-z, t) \\
& -4\left(1-\delta_{l n}\right) \partial_{x_{l}} \int_{\mathbb{R}^{n-1}}\left(\partial_{x_{i}} N\right)\left(\bar{x}-\bar{w}, x_{n}\right) \Gamma\left(\bar{w}-\bar{z}, z_{n}, t\right) d \bar{w} .
\end{aligned}
$$

Apply Proposition 2.2 for $i, l \neq n$, then we have

$$
G_{i l}(x, z, t)=\delta_{i l}\left(\Gamma(x-z, t)-\Gamma\left(x-z^{*}, t\right)\right)-\frac{2 \delta_{i l}}{n} \Gamma\left(x-z^{*}, t\right)
$$




$$
+4 \int_{0}^{x_{n}} \int_{\mathbb{R}^{n-1}} \partial_{x_{i}} \partial_{x_{l}} N(x-w) \Gamma\left(w-z^{*}, t\right) d w .
$$

Hence we have that for $i, l \neq n$,

$$
\begin{aligned}
& \partial_{x_{n}} \int_{\mathbb{R}_{+}^{n}} \partial_{y_{k}} \mathcal{G}(z, y) \partial_{z_{l}} G_{i l}(x, z, t) d z \\
= & \int_{\mathbb{R}_{+}^{n}} \partial_{y_{k}} \mathcal{G}(z, y) \partial_{z_{l}} \partial_{x_{n}} G_{i l}(x, z, t) d z \\
= & 4 \int_{\mathbb{R}_{+}^{n}} \partial_{y_{k}} \mathcal{G}(z, y) \partial_{z_{n}} \partial_{z_{l}}\left[\int_{0}^{x_{n}} \int_{\mathbb{R}^{n-1}} \partial_{x_{i}} \partial_{x_{l}} N(x-w) \Gamma\left(w-z^{*}, t\right) d w\right] d z \\
& -\delta_{i l} \int_{\mathbb{R}_{+}^{n}} \partial_{y_{k}} \mathcal{G}(z, y) \partial_{z_{l}} \partial_{z_{n}}\left[\Gamma(x-z, t)+\left(1+\frac{2}{n}\right) \Gamma\left(x-z^{*}, t\right)\right] d z \\
& -4 \int_{\mathbb{R}_{+}^{n}} \partial_{y_{k}} \mathcal{G}(z, y) \partial_{z_{l}}\left[\partial_{x_{l}} \int_{\mathbb{R}^{n-1}} \partial_{x_{i}} N\left(\bar{x}-\bar{w}, x_{n}\right) \Gamma\left(\bar{w}-\bar{z}, z_{n}, t\right) d \bar{w}\right] d z .
\end{aligned}
$$

Note that $\int_{\mathbb{R}_{+}^{n}} \partial_{y_{k}} \mathcal{G}(z, y) \partial_{z_{n}} f(z) d z=\partial_{y_{n}} \int_{\mathbb{R}_{+}^{n}} \partial_{y_{k}} \mathcal{G}^{-}(z, y) f(z) d z$, where

$$
\mathcal{G}^{-}(z, y) \equiv N(z-y)-N\left(z-y^{*}\right),
$$

since $\partial_{x_{n}}\left[\Gamma(x-z, t)-\Gamma\left(x-z^{*}, t\right)\right]=-\partial_{z_{n}}\left[\Gamma(x-z, t)+\Gamma\left(x-z^{*}, t\right)\right]$. Thus, we obtain that for $i, l \neq n$,

$$
\begin{aligned}
& \partial_{x_{n}} \int_{\mathbb{R}_{+}^{n}} \partial_{y_{k}} \mathcal{G}(z, y) \partial_{z_{l}} G_{i l}(x, z, t) d z \\
= & 4 \partial_{y_{n}} \int_{\mathbb{R}_{+}^{n}} \partial_{y_{k}} \mathcal{G}^{-}(z, y)\left[\int_{0}^{x_{n}} \int_{\mathbb{R}^{n-1}} \partial_{x_{i}} \partial_{x_{l}} N(x-w) \partial_{w_{l}}-\left(w-z^{*}, t\right) d w\right] d z \\
& -\delta_{i l} \partial_{y_{n}} \int_{\mathbb{R}_{+}^{n}} \partial_{y_{k}} \mathcal{G}^{-}(z, y) \partial_{z_{l}}\left[\Gamma(x-z, t)+\left(1+\frac{2}{n}\right) \Gamma\left(x-z^{*}, t\right)\right] d z \\
& -4 \partial_{y_{l}} \int_{\mathbb{R}_{+}^{n}} \partial_{y_{k}} \mathcal{G}(z, y)\left[\partial_{x_{l}} \int_{\mathbb{R}^{n-1}} \partial_{x_{i}} N\left(\bar{x}-\bar{w}, x_{n}\right) \Gamma\left(\bar{w}-\bar{z}, z_{n}, t\right) d \bar{w}\right] d z .
\end{aligned}
$$

Combining the above estimates (3.15) and (3.16), we obtain

$$
\begin{aligned}
\partial_{x_{n}} H_{i j k}(x, y, t)= & -\partial_{x_{n}} \partial_{y_{k}} G_{i j}(x, y, t) \\
& +\sum_{l=1}^{n-1} \partial_{y_{j}}\left(\tilde{H}_{i j k, l}^{1}(x, z, t)+\tilde{H}_{i j k, l}^{2}(x, y, t)+\tilde{H}_{i j k, l}^{3}(x, z, t)\right),
\end{aligned}
$$

where

$$
\begin{aligned}
& \tilde{H}_{i j k, l}^{1}(x, y, t) \\
\equiv & -\delta_{i l} \partial_{y_{n}} \int_{\mathbb{R}_{+}^{n}} \partial_{y_{k}} \mathcal{G}^{-}(z, y) \partial_{z_{l}}\left[\Gamma(x-z, t)+\left(1+\frac{2}{n}\right) \Gamma\left(x-z^{*}, t\right)\right] d z,
\end{aligned}
$$




$$
\begin{aligned}
& \tilde{H}_{i j k, l}^{2}(x, y, t) \\
\equiv & -4 \partial_{y_{n}} \int_{\mathbb{R}_{+}^{n}} \partial_{y_{k}} \mathcal{G}^{-}(z, y) \partial_{z_{l}}\left[\int_{0}^{x_{n}} \int_{\mathbb{R}^{n-1}} \partial_{x_{i}} \partial_{x_{l}} N(x-w) \Gamma\left(w-z^{*}, t\right) d w\right] d z, \\
& \tilde{H}_{i j k, l}^{3}(x, y, t) \\
\equiv & -4 \partial_{y_{l}} \int_{\mathbb{R}_{+}^{n}} \partial_{y_{k}} \mathcal{G}(z, y)\left[\partial_{x_{l}} \int_{\mathbb{R}^{n-1}} \partial_{x_{i}} N\left(\bar{x}-\bar{w}, x_{n}\right) \Gamma\left(\bar{w}-\bar{z}, z_{n}, t\right) d \bar{w}\right] d z .
\end{aligned}
$$

If $\mathcal{F}=\left(F_{j k}\right)=0$ on $x_{n}=0$, taking integrations by parts, $\partial_{x_{n}}\left(e^{-A t} \mathbb{P} \operatorname{div} \mathcal{F}\right)_{i}$ can be written by

$$
\begin{aligned}
& \partial_{x_{n}}\left(e^{-A t} \mathbb{P} \operatorname{div} \mathcal{F}\right)_{i}(x, t) \\
= & \int_{\mathbb{R}_{+}^{n}} \partial_{x_{n}} H_{i j k}(x, y, t) F_{j k}(y) d y \\
= & \int_{\mathbb{R}_{+}^{n}} \partial_{x_{n}} G_{i j}(x, y, t) \partial_{y_{k}} F_{j k}(y) d y \\
& -\sum_{l=1}^{n-1} \int_{\mathbb{R}_{+}^{n}}\left(\tilde{H}_{i j k, l}^{1}+\tilde{H}_{i j k, l}^{2}+\tilde{H}_{i j k, l}^{3}\right)(x, y, t) \partial_{y_{j}} F_{j k}(y) d y \\
= & \partial_{x_{n}}\left(e^{-t A} \operatorname{div} \mathcal{F}\right)_{i}(x)-\sum_{l=1}^{n-1} \int_{\mathbb{R}_{+}^{n}}\left(\tilde{H}_{i j k, l}^{1}+\tilde{H}_{i j k, l}^{2}+\tilde{H}_{i j k, l}^{3}\right)(x, y, t) \partial_{y_{j}} F_{j k}(y) d y .
\end{aligned}
$$

Hence

$$
\begin{aligned}
& \quad\left\|\partial_{x_{n}}\left(e^{-A t} \mathbb{P} \operatorname{div} \mathcal{F}\right)_{i}\right\|_{L^{\infty}} \\
& \leq C\left\|\partial_{x_{n}} e^{-A t} \operatorname{div} \mathcal{F}\right\|_{L^{\infty}}+C \max _{1 \leq j, k \leq n} \int_{\mathbb{R}_{+}^{n}}\left(\left|\tilde{H}_{i j k}^{1}(x, y, t)\right|+\left|\tilde{H}_{i j k}^{2}(x, y, t)\right|\right. \\
& \left.\quad+\left|\tilde{H}_{i j k}^{3}(x, y, t)\right|\right) d y\left\|\partial_{y_{k}} F_{j k}\right\|_{L^{\infty}} .
\end{aligned}
$$

From the known result (3.13) of Proposition 3.1, we have

$$
\left\|\partial_{x_{n}} e^{-A t} \operatorname{div} \mathcal{F}\right\|_{L^{\infty}} \leq C t^{-1 / 2}\|\operatorname{div} \mathcal{F}\|_{L^{\infty}} .
$$

Hence, we have only to estimate

$$
\int_{\mathbb{R}_{+}^{n}}\left(\left|\tilde{H}_{i j k}^{1}(x, y, t)\right|+\left|\tilde{H}_{i j k}^{2}(x, y, t)\right|+\left|\tilde{H}_{i j k}^{3}(x, y, t)\right|\right) d y
$$

for $i, l \neq n$. For the estimation, we will make use of the Hardy space theory.

First let us estimate $\int_{\mathbb{R}_{+}^{n}}\left|\tilde{H}_{i j k}^{1}(x, y, t)\right| d y$. Note that $\tilde{H}_{i j k}^{1}(x, y, t)$ is the linear sum of the terms

$$
\begin{aligned}
& \partial_{y_{n}} \int_{\mathbb{R}^{n}} \partial_{y_{k}} N(z-y) 1_{\mathbb{R}_{+}^{n}} \partial_{z_{l}} \Gamma(x-z, t) d z, \\
& \partial_{y_{n}} \int_{\mathbb{R}^{n}} \partial_{y_{k}} N(z-y) 1_{\mathbb{R}_{-}^{n}} \partial_{z_{l}} \Gamma(x-z, t) d z,
\end{aligned}
$$




$$
\partial_{y_{n}} \int_{\mathbb{R}^{n}} \partial_{y_{k}} N(z-y) 1_{\mathbb{R}_{+}^{n}} \partial_{z_{l}} \Gamma\left(x-z^{*}, t\right) d z,
$$

and

$$
\partial_{y_{n}} \int_{\mathbb{R}^{n}} \partial_{y_{k}} N(z-y) 1_{\mathbb{R}_{-}^{n}} \partial_{z_{l}} \Gamma\left(x-z^{*}, t\right) d z .
$$

Recall the well known result that $1_{\left\{z_{n}>0\right\}} \partial_{z_{l}} \Gamma(x-z, t), 1_{\left\{z_{n}<0\right\}} \partial_{z_{l}} \Gamma(x-z, t)$ are in Hardy space $\mathcal{H}^{1}\left(\mathbb{R}^{n}\right)$ for any fixed $x \in \mathbb{R}^{n}$. By the boundedness of the Calderon-Zygmund type transforms in Hardy spaces, we obtain that

$$
\begin{aligned}
\int_{\mathbb{R}_{+}^{n}}\left|\tilde{H}_{i j k}^{1}(x, y, t)\right| d y \leq & C\left\|1_{\mathbb{R}_{+}^{n}} \partial_{z_{i}} \Gamma(x-\cdot, t)\right\|_{\mathcal{H}^{1}}+C\left\|1_{\mathbb{R}_{-}^{n}} \partial_{z_{i}} \Gamma\left(x^{*}-\cdot, t\right)\right\|_{\mathcal{H}^{1}} \\
& +C\left\|1_{\mathbb{R}_{+}^{n}} \partial_{z_{i}} \Gamma\left(x^{*}-\cdot, t\right)\right\|_{\mathcal{H}^{1}}+C\left\|1_{\mathbb{R}_{-}^{n}} \partial_{z_{i}} \Gamma(x-\cdot, t)\right\|_{\mathcal{H}^{1}} \\
\leq & C t^{-1 / 2} .
\end{aligned}
$$

We rewrite $\tilde{H}_{i j k, l}^{2}$ by

$$
\begin{aligned}
& \tilde{H}_{i j k, l}^{2}(x, y, t) \\
= & \int_{0}^{x_{n}} \int_{\mathbb{R}^{n-1}} \frac{\partial^{2}}{\partial x_{n} \partial x_{i}} N(x-w)\left[\partial_{y_{l}} \int_{\mathbb{R}_{+}^{n}} \partial_{y_{k}} \mathcal{G}(z, y) \partial_{w_{l}} \Gamma\left(w-z^{*}, t\right) d z\right] d w .
\end{aligned}
$$

Set

$$
J_{k l}(w, y, t) \equiv \partial_{y_{l}} \int_{\mathbb{R}_{+}^{n}} \partial_{y_{k}} \mathcal{G}(z, y) \partial_{w_{l}} \Gamma\left(w-z^{*}, t\right) d z .
$$

By Lemma 2.7 we have

$$
\begin{aligned}
& \int_{\mathbb{R}_{+}^{n}}\left|\tilde{H}_{i j k, l}^{2}(x, y, t)\right| d y \\
\leq & C \sup _{y \in \mathbb{R}_{+}^{n}} \int_{\mathbb{R}_{+}^{n}}\left|J_{k l}(w, y, t)\right| d y \\
& +C x_{n}^{\alpha} \sup _{x_{n} / 2<z_{n}<x_{n}} \sup _{\bar{w} \in \mathbb{R}^{n-1}} \frac{\int_{\mathbb{R}_{+}^{n}}\left|J_{k l}(w, y, t)-J_{k l}\left(\bar{x}, w_{n}, y, t\right)\right| d y}{|\bar{x}-\bar{w}|^{\alpha}} .
\end{aligned}
$$

Note that

$$
\begin{aligned}
J_{k l}(w, y, t)= & \partial_{y_{l}} \int_{\mathbb{R}_{+}^{n}} \partial_{y_{k}}\left[N(z-y)+N\left(z-y^{*}\right)\right] \partial_{w_{l}} \Gamma\left(w-z^{*}, t\right) d z \\
= & \partial_{y_{l}} \int_{\mathbb{R}^{n}} \partial_{y_{k}} N(z-y) 1_{\left\{z_{n}>0\right\}} \partial_{w_{l}} \Gamma\left(w-z^{*}, t\right) d z \\
& +\partial_{y_{l}} \int_{\mathbb{R}^{n}} \partial_{y_{k}} N(z-y) 1_{\left\{z_{n}<0\right\}} \partial_{w_{l}} \Gamma(w-z, t) d z .
\end{aligned}
$$

Recall the well known result that $1_{\mathbb{R}_{+}^{n}} \partial_{w_{l}} \Gamma\left(w-z^{*}, t\right), 1_{\mathbb{R}_{-}^{n}} \partial_{w_{l}} \Gamma(w-z, t)$ are in the Hardy space $\mathcal{H}^{1}\left(\mathbb{R}^{n}\right)$ for any fixed $w \in \mathbb{R}^{n}$. Since Calderon-Zygmund type 
transforms are bounded in Hardy spaces, we obtain that

$$
\begin{aligned}
& \int_{\mathbb{R}_{+}^{n}}\left|J_{k l}(w, y, t)\right| d y \\
& \leq C\left\|1_{\mathbb{R}_{+}^{n}} \partial_{w_{l}} \Gamma\left(w^{*}-\cdot, t\right)\right\|_{\mathcal{H}^{1}}+\left\|1_{\mathbb{R}_{-}^{n}} \partial_{w_{l}} \Gamma(w-\cdot, t)\right\|_{\mathcal{H}^{1}} \leq C t^{-1 / 2} .
\end{aligned}
$$

Note that

$$
\begin{aligned}
& J_{k l}(w, y, t)-J_{k l}\left(\bar{x}, w_{n}, y, t\right) \\
= & \partial_{y_{l}} \int_{\mathbb{R}_{+}^{n}} \partial_{y_{k}}\left[N(z-y)+N\left(z-y^{*}\right)\right] \partial_{w_{l}}\left[\Gamma\left(w-z^{*}, t\right)-\Gamma\left(\bar{x}-\bar{z}, w_{n}+z_{n}, t\right)\right] d z .
\end{aligned}
$$

Recall Lemma 2.6 that for any $a=\left(\bar{w}, w_{n}\right), b=\left(\bar{x}, w_{n}\right) \in \mathbb{R}_{+}^{n}$,

$$
1_{\mathbb{R}_{+}^{n}}\left[\partial_{z_{l}}\left(\tau_{a^{*}} \Gamma_{t}\right)-\partial_{z_{l}}\left(\tau_{b^{*}} \Gamma_{t}\right)\right] \in \mathcal{H}^{1}\left(\mathbb{R}^{n}\right),
$$

of which norm is bounded by $C t^{-(1+\alpha) / 2} e^{-\frac{w_{n}^{2}}{4 t}}|\bar{w}-\bar{x}|^{\alpha}$ for $0<\alpha<1$. Hence by the same reasoning for $J_{k l}$, we conclude that

$$
\int_{\mathbb{R}_{+}^{n}}\left|J_{k l}(w, y, t)-J_{k l}\left(\bar{x}, w_{n}, y, t\right)\right| d y \leq C t^{-(1+\alpha) / 2} e^{-\frac{w_{n}^{2}}{4 t}}|\bar{w}-\bar{x}|^{\alpha} .
$$

Apply the estimates (3.18) and (3.19) to (3.17) we have

$$
\int_{\mathbb{R}_{+}^{n}}\left|\tilde{H}_{i j k, l}^{2}(x, y, t)\right| d y \leq C t^{-1 / 2}+C x_{n}^{\alpha} t^{-(1+\alpha) / 2} e^{-\frac{x_{n}^{2}}{4 t}} \leq C t^{-1 / 2} .
$$

Divide the domain of integration into two parts that

$$
\begin{aligned}
\tilde{H}_{i j k, l}^{3}(x, y, t)= & p . v \cdot \int_{|\bar{x}-\bar{w}| \leq \sqrt{t}} \partial_{x_{i}} N\left(\bar{x}-\bar{w}, x_{n}\right) J_{k l}((\bar{w}, 0), y, t) d \bar{w} \\
& +p \cdot v \cdot \int_{|\bar{x}-\bar{w}|>\sqrt{t}} \partial_{x_{i}} N\left(\bar{x}-\bar{w}, x_{n}\right) J_{k l}((\bar{w}, 0), y, t) d \bar{w} \\
\equiv & I+I I .
\end{aligned}
$$

By integrations by parts

$$
\begin{aligned}
I= & -p \cdot v \cdot \int_{|\bar{x}-\bar{w}| \leq \sqrt{t}} N\left(\bar{x}-\bar{w}, x_{n}\right) \partial_{y_{i}} J_{k l}((\bar{w}, 0), y, t) d \bar{w} \\
& +J_{k l}((\bar{x}, 0), y, t) \lim _{\epsilon \rightarrow 0} \int_{|\bar{x}-\bar{w}|=\epsilon} \frac{x_{i}-y_{i}}{\left|(\bar{x}-\bar{w})^{2}+x_{n}^{2}\right|^{(n-1) / 2}} d S_{\bar{w}} \\
& -\int_{|\bar{x}-\bar{w}|=\sqrt{t}} \frac{x_{i}-y_{i}}{|\bar{x}-\bar{w}|^{n-1}} J_{k l}((\bar{w}, 0), y, t) d S_{\bar{w}} .
\end{aligned}
$$

By the anti-symmetry, the second term is zero, hence we have

$$
I=-p \cdot v \cdot \int_{|\bar{x}-\bar{w}| \leq \sqrt{t}} N\left(\bar{x}-\bar{w}, x_{n}\right) \partial_{y_{i}} J_{k l}((\bar{w}, 0), y, t) d \bar{w}
$$




$$
\begin{aligned}
& -\int_{|\bar{x}-\bar{w}|=\sqrt{t}} \frac{x_{i}-y_{i}}{\left|(\bar{x}-\bar{w})^{2}+x_{n}^{2}\right|^{(n-1) / 2}} J_{k l}((\bar{w}, 0), y, t) d S_{\bar{w}} \\
\equiv & I_{1}+I_{2} .
\end{aligned}
$$

Recall the fact that $\partial_{y_{i}} J_{k l}$ is in Hardy space whose norm is bounded by $C t^{-1}$. If we integrate $I$ over $\mathbb{R}_{+}^{n}$ in terms of $y$ variables, then we have

$$
\begin{aligned}
\int_{\mathbb{R}_{+}^{n}}\left|I_{1}(x, y, t)\right| d y & \leq \int_{|\bar{x}-\bar{w}| \leq \sqrt{t}} \frac{d \bar{w}}{|\bar{x}-\bar{w}|^{n-2}} \sup _{\bar{w} \in \mathbb{R}^{n-1}} \int_{\mathbb{R}_{+}^{n}}\left|\partial_{y_{i}} J_{k l}((\bar{w}, 0), y, t)\right| d y \\
& \leq C \sqrt{t} \sup _{\bar{w} \in \mathbb{R}^{n-1}} \int_{\mathbb{R}_{+}^{n}}\left|\partial_{y_{i}} J_{k l}((\bar{w}, 0), y, t)\right| d y \leq C t^{-1 / 2},
\end{aligned}
$$

and

$$
\begin{aligned}
\int_{\mathbb{R}_{+}^{n}}\left|I_{2}(x, y, t)\right| d y & \leq \int_{|\bar{x}-\bar{w}|=\sqrt{t}} \frac{d S_{\bar{w}}}{|\bar{x}-\bar{w}|^{n-2}}\left(\sup _{\bar{w} \in \mathbb{R}^{n-1}} \int_{\mathbb{R}_{+}^{n}}\left|J_{k l}((\bar{w}, 0), y, t)\right| d y\right) \\
& \leq C \sup _{\bar{w} \in \mathbb{R}^{n-1}} \int_{\mathbb{R}_{+}^{n}}\left|J_{k l}((\bar{w}, 0), y, t)\right| d y \leq C t^{-1 / 2} .
\end{aligned}
$$

Set

$$
\tilde{J}_{k l}(\bar{w}, y, t) \equiv \partial_{y_{l}} \int_{\mathbb{R}_{+}^{n}} \partial_{y_{k}} \mathcal{G}(z, y) \Gamma((\bar{w}-\bar{z}, 0), t) d z .
$$

Note that $J_{k l}((\bar{w}, 0), y, t)=\partial_{w_{l}} \tilde{J}_{k l}(\bar{w}, y, t)$. Since $\partial_{w_{l}} \tilde{J}_{k l}(\bar{x}, y, t)=0$, by integration by parts, we obtain that

$$
\begin{aligned}
I I= & p \cdot v \cdot \int_{|\bar{x}-\bar{w}|>\sqrt{t}} \partial_{x_{i}} \partial_{x_{l}} N\left(\bar{x}-\bar{w}, x_{n}\right)\left[\tilde{J}_{k l}(\bar{w}, y, t)-\tilde{J}_{k l}(\bar{x}, y, t)\right] d \bar{w} \\
& -\lim _{R \rightarrow \infty} \int_{|\bar{x}-\bar{w}|=R} \frac{x_{i}-y_{i}}{|| \bar{x}-\left.\bar{w}\right|^{2}+\left.x_{n}^{2}\right|^{(n-1) / 2}} \frac{y_{l}-x_{l}}{|\bar{x}-\bar{y}|}\left[\tilde{J}_{k l}(\bar{w}, y, t)-\tilde{J}_{k l}(\bar{x}, y, t)\right] d S_{\bar{w}} \\
& +\int_{|\bar{x}-\bar{w}|=\sqrt{t}} \frac{x_{i}-y_{i}}{|| \bar{x}-\left.\bar{w}\right|^{2}+\left.x_{n}^{2}\right|^{(n-1) / 2}} \frac{y_{l}-x_{l}}{|\bar{x}-\bar{y}|}\left[\tilde{J}_{k l}(\bar{w}, y, t)-\tilde{J}_{k l}(\bar{x}, y, t)\right] d S_{\bar{w}} .
\end{aligned}
$$

Since

$$
\lim _{R \rightarrow \infty} \int_{|\bar{x}-\bar{w}|=R} \frac{R}{|| \bar{x}-\left.\bar{w}\right|^{2}+\left.x_{n}^{2}\right|^{n}} d S_{\bar{w}} \leq \lim _{R \rightarrow \infty} \frac{1}{R^{n-1}} \int_{|\bar{x}-\bar{w}|=R} d S_{\bar{w}}=0,
$$

we have

$$
\begin{aligned}
I I= & p \cdot v \cdot \int_{|\bar{x}-\bar{w}| \geq \sqrt{t}} \partial_{x_{i}} \partial_{x_{l}} N\left(\bar{x}-\bar{w}, x_{n}\right)\left[\tilde{J}_{k l}(\bar{w}, y, t)-\tilde{J}_{k l}(\bar{x}, y, t)\right] d \bar{w} \\
& +\int_{|\bar{x}-\bar{w}|=\sqrt{t}} \frac{\left(x_{i}-y_{i}\right)\left(x_{l}-y_{l}\right)}{\left|(\bar{x}-\bar{w})^{2}+x_{n}^{2}\right|^{(n+1) / 2}}\left[\tilde{J}_{k l}(\bar{w}, y, t)-\tilde{J}_{k l}(\bar{x}, y, t)\right] d S_{\bar{w}} \\
\equiv & I I_{1}+I I_{2} .
\end{aligned}
$$

If we integrate $I I$ over $\mathbb{R}_{+}^{n}$ in terms of $y$ variables, then we have

$$
\int_{\mathbb{R}_{+}^{n}}\left|I I_{1}(x, y, t)\right| d y
$$




$$
\begin{aligned}
& \leq \int_{|\bar{x}-\bar{w}| \geq \sqrt{t}} \frac{d \bar{w}}{|\bar{x}-\bar{w}|^{n-\alpha}} \sup _{\bar{w} \in \mathbb{R}^{n-1}} \frac{\int_{\mathbb{R}_{+}^{n}}\left|\tilde{J}_{k l}(\bar{w}, y, t)-\tilde{J}_{k l}(\bar{x}, y, t)\right| d y}{|\bar{x}-\bar{w}|^{\alpha}} \\
& \leq C\left(\sqrt{t}+x_{n}\right)^{-1+\alpha} \sup _{\bar{w} \in \mathbb{R}^{n-1}} \frac{\int_{\mathbb{R}_{+}^{n}}\left|\tilde{J}_{k l}(\bar{w}, y, t)-\tilde{J}_{k l}(\bar{x}, y, t)\right| d y}{|\bar{x}-\bar{w}|^{\alpha}}
\end{aligned}
$$

and

$$
\begin{aligned}
& \int_{\mathbb{R}_{+}^{n}}\left|I I_{2}(x, y, t)\right| d y \\
\leq & \int_{|\bar{x}-\bar{w}|=\sqrt{t}} \frac{d S_{\bar{w}}}{|\bar{x}-\bar{w}|^{n-1-\alpha}} \sup _{\bar{w} \in \mathbb{R}^{n-1}} \frac{\int_{\mathbb{R}_{+}^{n}}\left|\tilde{J}_{k l}(\bar{w}, y, t)-\tilde{J}_{k l}(\bar{x}, y, t)\right| d y}{|x-\bar{w}|^{\alpha}} \\
\leq & C\left(\sqrt{t}+x_{n}\right)^{-1+\alpha} \sup _{\bar{w} \in \mathbb{R}^{n-1}} \frac{\int_{\mathbb{R}_{+}^{n}}\left|\tilde{J}_{k l}(\bar{w}, y, t)-\tilde{J}_{k l}(\bar{x}, y, t)\right| d y}{|x-\bar{w}|^{\alpha}} .
\end{aligned}
$$

Now recall that $\Gamma\left(\bar{w}-\bar{z}, z_{n}, t\right)-\Gamma\left(\bar{x}-\bar{z}, z_{n}, t\right) \in \mathcal{H}^{1}\left(\mathbb{R}_{+}^{n}\right)$ for any fixed $\bar{w}, \bar{x} \in$ $\mathbb{R}^{n-1}$ whose norm is bounded by $C t^{-\alpha / 2}$ and $\tilde{J}_{k l}$ is linear sum of the terms

$$
\begin{gathered}
\partial_{y_{n}} \int_{\mathbb{R}^{n}} \partial_{y_{k}} N(z-y) 1_{\mathbb{R}_{+}^{n}} \partial_{z_{l}}\left[\Gamma\left(\bar{w}-\bar{z}, z_{n}, t\right)-\Gamma\left(\bar{x}-\bar{z}, z_{n}, t\right)\right] d z, \\
\partial_{y_{n}} \int_{\mathbb{R}^{n}} \partial_{y_{k}} N(z-y) 1_{\mathbb{R}_{-}^{n}}\left[\Gamma\left(\bar{w}-\bar{z}, z_{n}, t\right)-\Gamma\left(\bar{x}-\bar{z}, z_{n}, t\right)\right] d z .
\end{gathered}
$$

By the boundedness of Calderon-Zygmund type transforms in Hardy spaces, we obtain that

$$
\int_{\mathbb{R}_{+}^{n}}\left|\tilde{J}_{k l}(\bar{w}, y, t)-\tilde{J}_{k l}(\bar{x}, y, t)\right| d y \leq C t^{-\alpha / 2}|\bar{x}-\bar{w}|^{\alpha}, \quad 0<\alpha<1 .
$$

Hence we have

$$
\int_{\mathbb{R}_{+}^{n}}|I I(x, y, t)| d y \leq C t^{-1 / 2} .
$$

Therefore we conclude that:

\section{Lemma 3.4.}

$$
\left\|\partial_{x_{n}} \overline{e^{-t A} \mathbb{P} \operatorname{div} \mathcal{F}}\right\|_{L^{\infty}\left(\mathbb{R}_{+}^{n}\right)} \leq C t^{-1 / 2}\left\|\partial_{x_{n}} \mathcal{F}\right\|_{L^{\infty}\left(\mathbb{R}_{+}^{n}\right)} .
$$

Combining Lemma 3.2, Lemma 3.3 and Lemma 3.4, we have the following estimate for $\nabla e^{-t A} \mathbb{P} \operatorname{div} \mathcal{F}$.

Theorem 3.5.

$$
\left\|\nabla e^{-t A} \mathbb{P} \operatorname{div} \mathcal{F}\right\|_{L^{\infty}\left(\mathbb{R}_{+}^{n}\right)} \leq C t^{-1 / 2}\|\nabla \mathcal{F}\|_{L^{\infty}\left(\mathbb{R}_{+}^{n}\right)} .
$$




\section{Proof of Theorem 1.2}

Set $u_{1}=e^{-t A} a$ and $u_{0}=0$. For given $u_{n-1}$, define $u_{n}$ recursively by

$$
u_{n}=e^{-t A} a+B\left(u_{n-1}, u_{n-1}\right) .
$$

In [16], it is well known that

$$
\left\|e^{-t A} a\right\|_{\infty} \leq C_{0}\|a\|_{\infty},
$$

and from Proposition 3.1 we have the estimate

$$
\left\|e^{-(t-s) A} \mathbb{P} \operatorname{div}\left(u_{n-1} \otimes u_{n-1}\right)\right\|_{\infty} \leq C_{1}(t-s)^{-\frac{1}{2}}\left\|\left(u_{n-1} \otimes u_{n-1}\right)\right\|_{\infty} .
$$

Hence we have the following estimate for $\left\|u_{n}\right\|_{\infty}$.

$$
\left\|u_{n}\right\|_{\infty} \leq C_{0}\left\|u_{0}\right\|_{\infty}+C_{1} \int_{0}^{t}(t-s)^{-\frac{1}{2}}\left\|u_{n-1}(s)\right\|_{\infty}^{2} d s .
$$

Take large $M \geq 2 C_{0}\|a\|_{L^{\infty}}$. Suppose $\sup _{0<t<T}\left\|u_{n-1}(t)\right\|_{\infty} \leq M$, then from (4.20) we have

$$
\begin{aligned}
\left\|u_{n}(t)\right\|_{\infty} & \leq C_{0}\left\|u_{0}\right\|_{\infty}+C_{1}\left[\int_{0}^{t}(t-s)^{-\frac{1}{2}} d s\right]\left[\sup _{0<s<t}\left\|u_{n-1}(t)\right\|_{\infty}\right]^{2} \\
& \leq \frac{M}{2}+C_{1} C_{2} \sqrt{t} M^{2}
\end{aligned}
$$

where $\int_{0}^{t}(t-s)^{-\frac{1}{2}} d s=C_{2} \sqrt{t}$. Hence we have the inequality

$$
\sup _{0<t<T}\left\|u_{n}(t)\right\|_{\infty} \leq \frac{M}{2}+C_{1} C_{2} \sqrt{T} M^{2} .
$$

Take $T>0$ small enough that $2 C_{1} C_{2} \sqrt{T} M<1$, then we have

$$
\sup _{0<t<T}\left\|u_{n}(t)\right\|_{\infty} \leq M .
$$

By (3.13) of Proposition 3.1, we have the estimate

$$
\left\|\nabla e^{-t A} a\right\|_{\infty} \leq C_{3} t^{-1 / 2}\|a\|_{\infty}
$$

and by Theorem 3.5 , we have the estimate

$$
\left\|\nabla e^{-(t-s) A} \mathbb{P} \operatorname{div}\left(u_{n-1} \otimes u_{n-1}\right)\right\|_{\infty} \leq C_{4}(t-s)^{-\frac{1}{2}}\left\|\nabla\left(u_{n-1} \otimes u_{n-1}\right)\right\|_{\infty} .
$$

Hence we have the following estimate for $\left\|\nabla u_{n}\right\|_{L^{\infty}\left(\mathbb{R}_{+}^{n}\right)}$.

$$
\left\|\nabla u_{n}\right\|_{\infty} \leq C_{3} t^{-1 / 2}\left\|u_{0}\right\|_{\infty}+\int_{0}^{t}(t-s)^{-\frac{1}{2}} C_{4}\left\|u_{n-1}(s)\right\|_{\infty}\left\|\nabla u_{n-1}(s)\right\|_{\infty} d s .
$$

Take large $M_{1} \geq 2 C_{3}\|a\|_{\infty}$. Suppose $\sup _{0<t<T} t^{1 / 2}\left\|\nabla u_{n-1}(t)\right\|_{\infty} \leq M_{1}$, then from (4.21) we have

$$
\left\|\nabla u_{n}(t)\right\|_{\infty} \leq C_{3} t^{-1 / 2}\left\|u_{0}\right\|_{\infty}
$$




$$
\begin{aligned}
& +C_{4}\left[\int_{0}^{t}(t-s)^{-\frac{1}{2}} s^{-1 / 2} d s\right]\left[\sup _{0<s<t}\left\|u_{n-1}(s)\right\|_{\infty}\right]\left[\sup _{0<s<t} s^{1 / 2}\left\|\nabla u_{n-1}(s)\right\|_{\infty}\right] \\
\leq & t^{-1 / 2} \frac{M_{1}}{2}+C_{4} C_{5} M M_{1},
\end{aligned}
$$

where $\int_{0}^{t}(t-s)^{-\frac{1}{2}} s^{-1 / 2} d s=C_{5}$. Hence we have the inequality

$$
\sup _{0<t<T} t^{1 / 2}\left\|\nabla u_{n}\right\|_{\infty} \leq \frac{M_{1}}{2}+C_{4} C_{5} M M_{1} \sqrt{T}
$$

Take $T>0$ small enough that $4 C_{4} C_{5} M \sqrt{T}<1$, then we have

$$
\sup _{0<t<T} t^{1 / 2}\left\|\nabla u_{n}(t)\right\|_{\infty} \leq M_{1} .
$$

This implies that $\left\{u_{n}\right\}_{n=1}^{\infty}$ is uniformly bounded in $L^{\infty}\left((0, T) ; W^{1, \infty}\left(\mathbb{R}_{+}^{n}\right)\right)$, if $T$ is so small that

$$
2 C_{1} C_{2} \sqrt{T} M<1, \quad 4 C_{4} C_{5} M \sqrt{T}<1,
$$

where $M=2 C_{0}\|a\|_{\infty}$ and $M_{1}=2 C_{3}\|a\|_{\infty}$.

Set $w_{n}=u_{n+1}-u_{n}, n=1,2, \ldots$. Note that

$$
\sup _{0<t<T}\left\|w_{0}(t)\right\|_{\infty}=\sup _{0<t<T}\left\|e^{-t A} u_{0}\right\|_{\infty} \leq C_{0}\|a\|_{\infty} .
$$

Note

$$
B\left(u_{n}, u_{n}\right)-B\left(u_{n-1}, u_{n-1}\right)=B\left(u_{n}, u_{n}-u_{n-1}\right)+B\left(u_{n}-u_{n-1}, u_{n-1}\right) .
$$

Then we also have the following estimate for $\left\|w_{n}\right\|_{\infty}$.

$$
\left\|w_{n}(t)\right\|_{\infty} \leq C_{1} \int_{0}^{t}(t-s)^{-\frac{1}{2}}\left(\left\|u_{n}(s)\right\|_{\infty}+\left\|u_{n-1}(s)\right\|_{\infty}\right)\left\|w_{n-1}(s)\right\|_{\infty} d s .
$$

Recall that if $M \geq 2 C_{0}\|a\|_{L^{\infty}\left(\mathbb{R}_{+}^{n}\right)}$ and $2 C_{1} C_{2} M \sqrt{T}<1$, then

$$
\sup _{0<t<T}\left\|u_{n}\right\|_{L^{\infty}\left(\mathbb{R}_{+}^{n}\right)} \leq M
$$

for all $n=1,2, \ldots$. From (4.22) we have

$$
\begin{aligned}
\sup _{0<t<T}\left\|w_{n}(t)\right\|_{\infty} & \leq 2 C_{1} C_{2} M \sqrt{T} \sup _{0<t<T}\left\|w_{n-1}(t)\right\|_{\infty} \\
& \leq\left(2 C_{1} C_{2} M \sqrt{T}\right)^{n} C_{0}\|a\|_{\infty} .
\end{aligned}
$$

Since $2 C_{1} C_{2} M \sqrt{T}<1$, the series $\sum_{n=1}^{\infty} w_{n}(t)$ converges to some $u \in$ $L^{\infty}\left((0, T) \times \mathbb{R}_{+}^{n}\right)$. This implies $u_{n}$ converges to some $u$ in $L^{\infty}\left((0, T) \times \mathbb{R}_{+}^{n}\right)$, since $\sum_{k=0}^{n-1} w_{n}(t)=u_{n}(t)$. By the lower semi-continuity, we have

$$
\sup _{0<t<T}\|u(t)\|_{\infty} \leq M, \quad \sup _{0<t<T} t^{1 / 2}\|\nabla u(t)\|_{\infty} \leq M_{1} .
$$




\section{Proof of Theorem $\mathbf{1 . 3}$}

Let $u$ and $v$ be the mild solution of the Navier-Stokes equations in the class $L_{\mathrm{loc}}^{\infty}\left(0, T ; L^{\infty}\left(\mathbb{R}_{+}^{n}\right)\right)$ with the property

$$
\sup _{0<t<T} t^{\frac{\alpha}{2}}\|u(t)\|_{\infty}=M_{1} \text { and } \sup _{0<t<T} t^{\frac{\alpha}{2}}\|u(t)\|_{\infty}=M_{2} .
$$

Here, $0<\alpha<1$

Set $w=u-v$, then $w$ satisfies the integral equation

$$
w=B(u, u)-B(v, v)=B(w, u)+B(v, w) .
$$

Apply Proposition 3.1, then we obtain the following Gronwall inequality

$$
\begin{aligned}
\|w(t)\|_{\infty} & \leq C_{1} \int_{0}^{t}(t-s)^{-\frac{1}{2}}\left(\|u(s)\|_{\infty}+\|v(s)\|_{\infty}\right)\|w(s)\|_{\infty} d s \\
& \leq C_{1}\left(M_{1}+M_{2}\right) \int_{0}^{t}(t-s)^{-\frac{1}{2}} s^{-\frac{\alpha}{2}}\|w(s)\|_{\infty} d s .
\end{aligned}
$$

Set $X(t)=\|w(t)\|_{\infty}$. Then $X$ satisfies inequality

$$
X(t) \leq C_{1}\left(M_{1}+M_{2}\right)\left[\int_{0}^{t}(t-s)^{-\frac{1}{2}} s^{-\frac{\alpha}{2}} X(s) d s\right] .
$$

Note that $\int_{0}^{t}(t-s)^{-\frac{1}{2}} s^{-\alpha} d s=C_{2} t^{\frac{1}{2}-\alpha}$ if $0 \leq \alpha<1$. Then

$$
\sup _{0<t \leq t_{0}} t^{\frac{\alpha}{2}} X(t) \leq C_{1} C_{2}\left(M_{1}+M_{2}\right) t_{0}^{\frac{1}{2}-\frac{\alpha}{2}}\left(\sup _{0<s \leq t_{0}} s^{\frac{\alpha}{2}} X(s)\right) .
$$

Choose $t_{0}$ small enough that $C_{1} C_{2}\left(M_{1}+M_{2}\right) t_{0}^{\frac{1}{2}-\frac{\alpha}{2}}<1$, then (5.25) implies that

$$
\sup _{0<t \leq t_{0}} t^{\frac{\alpha}{2}} X(t)=0
$$

This again implies that $X(t)=0$ for all $0<t \leq t_{0}$. Then from (5.24) we have

$$
\begin{aligned}
X(t) & \leq C_{1}\left(M_{1}+M_{2}\right)\left[\int_{t_{0}}^{t}(t-s)^{-\frac{1}{2}} s^{-\frac{\alpha}{2}} X(s) d s\right] \\
& \leq C_{1}\left(M_{1}+M_{2}\right) t_{0}^{-\frac{\alpha}{2}} \int_{t_{0}}^{t}(t-s)^{-\frac{1}{2}} X(s) d s .
\end{aligned}
$$

Iterating the estimate (5.26), we obtain

$$
\begin{aligned}
X(t) & \leq C_{1}^{2}\left(M_{1}+M_{2}\right)^{2} t_{0}^{-\alpha} \int_{t_{0}}^{t}(t-s)^{-\frac{1}{2}}\left[\int_{t_{0}}^{s}(s-\tau)^{-\frac{1}{2}} X(\tau) d \tau\right] d s \\
& =C_{1}^{2}\left(M_{1}+M_{2}\right)^{2} t_{0}^{-\alpha} \int_{t_{0}}^{t}\left[\int_{\tau}^{t}(t-s)^{-\frac{1}{2}}(s-\tau)^{-\frac{1}{2}} d s\right] X(\tau) d \tau \\
& \leq C_{1}^{2} C_{3}\left(M_{1}+M_{2}\right)^{2} t_{0}^{-\alpha} \int_{t_{0}}^{t} X(\tau) d \tau .
\end{aligned}
$$

Here, we noted that $\int_{\tau}^{t}(t-s)^{-\frac{1}{2}}(s-\tau)^{-\frac{1}{2}} d s=C_{3}<\infty$. 
Set $Y(t)=\int_{t_{0}}^{t} X(\tau) d \tau$. Then from (5.27) we have the Gronwall inequality

$$
Y^{\prime}(t) \leq C_{1}^{2} C_{3}\left(M_{1}+M_{2}\right)^{2} t_{0}^{-\alpha} Y(t) \text { and } Y\left(t_{0}\right)=0 \text {. }
$$

Solving the above Gronwall inequality we conclude that $Y(t) \equiv 0$ for $t_{0}<t<T$. Applying this result to (5.27), then we have $X(t) \leq C_{1}^{2} C_{3}\left(M_{1}+M_{2}\right)^{2} t_{0}^{-\alpha} Y(t)=$ 0 for $t_{0}<t<T$.

Therefore we conclude that $\|w(t)\|_{\infty}=X(t)=0$ for all $0<t<T$, that is, $u(t)=v(t)$ for all $0<t<T$.

\section{Appendix A. Proof of Proposition 2.2.}

Let $x \in \mathbb{R}_{+}^{n}$. Choose a nonnegative smooth function $\phi \in C_{0}^{\infty}([0,2))$ with $\phi(t)=1$ for $0 \leq t \leq 1$ and $\int_{0}^{2} \phi(t) d t=1$. For $\epsilon<\frac{x_{n}}{2}$, define a cut-off function $\phi_{\epsilon, x}$ by $\phi_{\epsilon, x}(z)=\phi\left(\frac{|x-z|}{\epsilon}\right)$. Then $\phi_{\epsilon, x}$ is compactly supported in $\mathbb{R}_{+}^{n}$.

Define a function $v_{\epsilon}$ by

$$
v_{\epsilon}(x)=\int_{0}^{x_{n}} \int_{\mathbb{R}^{n-1}} \phi_{\epsilon, x}(z) \partial_{z_{i}} N(x-z) f(z) d z .
$$

We differentiate $v_{\epsilon}$ by $x_{j}$ variable. If $j \neq n$, we have

$\partial_{x_{j}} v_{\epsilon}(x)=\int_{0}^{x_{n}} \int_{\mathbb{R}^{n-1}}\left[\partial_{x_{j}} \phi_{\epsilon, x}(z) \partial_{z_{i}} N(x-z)+\phi_{\epsilon, x}(z) \partial_{z_{i}} \partial_{x_{j}} N(x-z)\right] f(z) d z$.

If $j=n$, we have

$$
\begin{aligned}
\partial_{x_{n}} v_{\epsilon}(x)= & \int_{0}^{x_{n}} \int_{\mathbb{R}^{n-1}}\left[\partial_{x_{n}} \phi_{\epsilon, x}(z) \partial_{z_{i}} N(x-z)+\phi_{\epsilon, x}(z) \partial_{z_{i}} \partial_{x_{n}} N(x-z)\right] f(z) d z \\
& +\int_{\mathbb{R}^{n-1}} \phi_{\epsilon, x}\left(\bar{z}, x_{n}\right) \partial_{x_{i}} N(\bar{x}-\bar{z}, 0) f\left(\bar{z}, x_{n}\right) d \bar{z}
\end{aligned}
$$

Set

$$
\begin{aligned}
I_{\epsilon}(x) & =\int_{0}^{x_{n}} \int_{\mathbb{R}^{n-1}} \partial_{x_{j}} \phi_{\epsilon, x}(z) \partial_{x_{i}} N(x-z) f(z) d z, \quad \text { and } \\
I I_{\epsilon}(x) & =\int_{0}^{x_{n}} \int_{\mathbb{R}^{n-1}} \phi_{\epsilon, x}(z) \frac{\partial^{2}}{\partial x_{i} \partial x_{j}} N(x-z) f(z) d z .
\end{aligned}
$$

Obviously,

$$
I I_{\epsilon}(x) \rightarrow \int_{0}^{x_{n}} \int_{\mathbb{R}^{n-1}} \frac{\partial^{2}}{\partial x_{i} \partial x_{j}} N(x-z) f(z) d z \quad \text { as } \epsilon \rightarrow 0 .
$$

Divide $I_{\epsilon}$ by $I_{\epsilon}^{1}$ and $I_{\epsilon}^{2}$ defined by

$$
I_{\epsilon}^{1}(x)=\int_{0}^{x_{n}} \int_{\mathbb{R}^{n-1}} \partial_{x_{j}} \phi_{\epsilon, x}(z) \partial_{x_{i}} N(x-z)[f(z)-f(x)] d z
$$

and

$$
I_{\epsilon}^{2}(x)=f(x) \int_{0}^{x_{n}} \int_{\mathbb{R}^{n-1}} \partial_{x_{j}} \phi_{\epsilon, x}(z) \partial_{x_{i}} N(x-z) d z
$$


Note that $I_{\epsilon}^{1}(x) \rightarrow 0$ as $\epsilon \rightarrow 0$, since

$$
\begin{aligned}
\left|I_{\epsilon}^{1}(x)\right| & \leq \int_{|x-z| \leq \epsilon}\left|\partial_{x_{j}} \phi_{\epsilon, x}(z)\right|\left|\partial_{x_{i}} N(x-z)\right||f(z)-f(x)| d z \\
& \leq \int_{|x-z| \leq \epsilon} \frac{1}{\epsilon} \frac{1}{|x-z|^{n-1}}|x-z|^{\alpha} d z[f]_{\alpha} \leq \epsilon^{\alpha}[f]_{\alpha},
\end{aligned}
$$

where

$$
[f]_{\alpha}=\sup _{x, z,|x-z| \leq \epsilon} \frac{|f(x)-f(z)|}{|x-z|^{\alpha}} .
$$

Now it remains to show that

$$
\int_{0}^{x_{n}} \int_{\mathbb{R}^{n-1}} \partial_{x_{j}} \phi_{\epsilon, x}(z) \partial_{x_{i}} N(x-z) d z=\left\{\begin{array}{l}
-\frac{\delta_{i j}}{2 n} \text { if } i, j \neq n \text { or } i=j=n \\
0 \text { if } i=n, j \neq n \text { or } j=n, i \neq n .
\end{array}\right.
$$

Taking change of variables and sending $\epsilon$ to the zero, we obtain

$$
\begin{aligned}
& \int_{0}^{x_{n}} \int_{\mathbb{R}^{n-1}} \partial_{x_{j}} \phi_{\epsilon, x}(z) \partial_{x_{i}} N(x-z) d z \\
= & \frac{1}{\epsilon} \int_{0}^{x_{n}} \int_{\mathbb{R}^{n-1}} \frac{\left(z_{i}-x_{j}\right)\left(z_{j}-x_{j}\right)}{n \omega_{n}|x-z|^{n+1}} \phi^{\prime}\left(\frac{|x-z|}{\epsilon}\right) d z \\
\rightarrow & \int_{0}^{\infty} \int_{\mathbb{R}^{n-1}} \frac{w_{i} w_{j}}{n \omega_{n}|w|^{n+1}} \phi^{\prime}(|w|) d w .
\end{aligned}
$$

If $i, j \neq n$ or $i=j=n$, then by the symmetry of $\frac{w_{i} w_{j}}{n \omega_{n}|w|^{n+1}} \phi^{\prime}(|w|)$ in terms of $w_{n}$ variables, we have

$$
\begin{aligned}
& \int_{0}^{\infty} \int_{\mathbb{R}^{n-1}} \frac{w_{i} w_{j}}{n \omega_{n}|w|^{n+1}} \phi^{\prime}(|w|) d w=\frac{1}{2} \int_{\mathbb{R}^{n}} \frac{w_{i} w_{j}}{n \omega_{n}|w|^{n}} \phi^{\prime}(|w|) d w \\
= & \frac{1}{2 n \omega_{n}} \int_{0}^{2} \int_{s^{n-1}} r w_{i} w_{j} \phi^{\prime}(r) d S_{w} d r=-\frac{1}{2 n} \delta_{i j} \int_{0}^{2} \phi(r) d r=-\frac{1}{2 n} \delta_{i j} .
\end{aligned}
$$

If $i=n, j \neq n$ or $j=n, i \neq n$, then by the anti-symmetry of $\frac{w_{i} w_{j}}{n \omega_{n}|w|^{n+1}} \phi^{\prime}(|w|)$ in terms of $w_{n}$ variables, we have

$$
\int_{0}^{\infty} \int_{\mathbb{R}^{n-1}} \frac{w_{i} w_{j}}{n \omega_{n}|w|^{n+1}} \phi^{\prime}(|w|) d w=0
$$

\section{Appendix B. Proof of Lemma 2.4}

Let $k=1, \ldots, n-1$. Set $f_{t}(x)=f(x, t)=1_{\mathbb{R}_{+}^{n}} \partial_{x_{k}}\left(\tau_{a} \Gamma_{t}\right)$. We would like to show that $\mathcal{N} f_{t}(x) \in L^{1}\left(\mathbb{R}^{n}\right)$. For $s>0$, we have

$$
\begin{aligned}
& f_{t} * \Gamma_{s}(x) \\
= & \int_{\mathbb{R}^{n}} f(x-y, t) \Gamma_{s}(y) d y \\
= & \int_{\mathbb{R}^{n-1}} \int_{-\infty}^{x_{n}} \partial_{x_{k}} \Gamma(a-x+y, t) \Gamma(y, s) d y
\end{aligned}
$$




$$
\begin{aligned}
& =\left[\int_{\mathbb{R}^{n-1}} \partial_{x_{k}} \bar{\Gamma}(\bar{a}-\bar{x}+\bar{y}, t) \bar{\Gamma}(\bar{y}, s) d \bar{y}\right]\left[\int_{-\infty}^{x_{n}} \Gamma_{t}^{n}\left(a_{n}-x_{n}+y_{n}\right) \Gamma_{s}^{n}\left(y_{n}\right) d y_{n}\right] \\
& =\left(\partial_{x_{k}} \bar{\Gamma}_{s+t}\right)(\bar{a}-\bar{x})\left[\int_{-\infty}^{x_{n}} \Gamma_{t}^{n}\left(a_{n}-x_{n}+y_{n}\right) \Gamma_{s}^{n}\left(y_{n}\right) d y_{n}\right] .
\end{aligned}
$$

Note that

$$
\left|\partial_{x_{k}} \bar{\Gamma}_{s+t}(\bar{a}-\bar{x})\right| \leq C(s+t)^{-\frac{1}{2}} \bar{\Gamma}_{2(s+t)}(\bar{a}-\bar{x}),
$$

and

$$
\begin{aligned}
\int_{-\infty}^{x_{n}} \Gamma_{t}^{n}\left(a_{n}-x_{n}+y_{n}\right) \Gamma_{s}^{n}\left(y_{n}\right) d y_{n} & \leq \int_{-\infty}^{\infty} \Gamma_{t}^{n}\left(a_{n}-x_{n}+y_{n}\right) \Gamma_{s}^{n}\left(y_{n}\right) d y_{n} \\
& =\Gamma_{s+t}^{n}\left(a_{n}-x_{n}\right) .
\end{aligned}
$$

Hence we obtain that

$$
\left|f_{t} * \Gamma_{s}(x)\right| \leq C(s+t)^{-\frac{1}{2}} \Gamma_{2(t+s)}(a-x) .
$$

Note that $\Gamma_{2(t+s)}(a-x) \leq C(s+t)^{-n / 2}$ and $\Gamma_{2(t+s)}(a-x) \leq C(s+t)^{\frac{1}{2}} \mid a-$ $\left.x\right|^{-n-1}$. Hence we have that

$$
\begin{aligned}
\mathcal{N} f_{t}(x)=\sup _{s>0}\left|\Gamma_{s} * f_{t}(x)\right| & \leq \sup _{s>0} \min \left\{(s+t)^{-\frac{n+1}{2}},|x-a|^{-n-1}\right\} \\
& \leq \min \left\{t^{-\frac{n+1}{2}},|x-a|^{-n-1}\right\} .
\end{aligned}
$$

Now we estimate the $L^{1}$ norm of $\mathcal{N} f_{t}$.

$$
\begin{aligned}
\int_{\mathbb{R}^{n}} \mathcal{N} f_{t}(x) d x & =\int_{|x-a| \geq \sqrt{t}} \mathcal{N} f_{t}(x) d x+\int_{|x-a| \leq \sqrt{t}} \mathcal{N} f_{t}(x) d x \\
& \leq \int_{|x-a| \geq \sqrt{t}}|x-a|^{-n-1} d x+\int_{|x-a| \leq \sqrt{t}} t^{-\frac{n+1}{2}} d x \\
& \leq C t^{-\frac{1}{2}} .
\end{aligned}
$$

This leads to the conclusion that

$$
f_{t}=1_{\mathbb{R}_{+}^{n}} \tau_{a} \partial_{y_{k}} \Gamma_{t} \in \mathcal{H}^{1}\left(\mathbb{R}^{n}\right) .
$$

\section{Appendix C. Proof of Lemma 2.6}

Let $a=\left(\bar{a}, a_{n}\right)$ and $b=\left(\bar{b}, a_{n}\right)$. Set

$$
f_{t}(x)=f(x, t)=1_{\mathbb{R}_{+}^{n}}\left[\left(\tau_{a^{*}} \Gamma_{t}\right)(x)-\left(\tau_{b^{*}} \Gamma_{t}\right)(x)\right] .
$$

We would like to estimate that the $L^{1}$ norm of $\mathcal{N} f_{t}(x)=\sup _{s>0}\left|f_{t} * \Gamma_{s}(x)\right|$. In the proof of Lemma 2.4, we recall that

$$
\left(1_{\mathbb{R}_{+}^{n}} \Gamma_{t}^{a^{*}}\right) * \Gamma_{s}(x)=\bar{\Gamma}_{t+s}(\bar{a}-\bar{x})\left[\int_{-\infty}^{x_{n}} \Gamma_{t}^{n}\left(a_{n}+x_{n}-y_{n}\right) \Gamma_{s}^{n}\left(y_{n}\right) d y_{n}\right] .
$$

Hence we have that

$$
f_{t} * \Gamma_{s}(x)=\left[\bar{\Gamma}_{t+s}(\bar{a}-\bar{x})-\bar{\Gamma}_{t+s}(\bar{b}-\bar{x})\right]\left[\int_{-\infty}^{x_{n}} \Gamma_{t}^{n}\left(a_{n}+x_{n}-y_{n}\right) \Gamma_{s}^{n}\left(y_{n}\right) d y_{n}\right] .
$$


Recall that

$$
\int_{-\infty}^{x_{n}} \Gamma_{t}^{n}\left(a_{n}+x_{n}-y_{n}\right) \Gamma_{s}^{n}\left(y_{n}\right) d y_{n} \leq C e^{-\frac{a_{n}^{2}}{4 t}} \Gamma_{s+t}^{n}\left(x_{n}\right) .
$$

If $|\bar{a}-\bar{b}| \leq \frac{|\bar{x}-\bar{a}|}{2}$, then by the mean value theorem we have that

$$
\begin{aligned}
& \left|\bar{\Gamma}_{t+s}(\bar{a}-\bar{x})-\bar{\Gamma}_{t+s}(\bar{b}-\bar{x})\right| \\
\leq & |\bar{b}-\bar{a}| \mid \nabla_{\bar{x}} \bar{\Gamma}_{s+t}(\bar{a}-\bar{x}+\theta(\bar{b}-\bar{a}) \mid \\
\leq & C(s+t)^{-1 / 2}|\bar{b}-\bar{a}| \bar{\Gamma}_{4(s+t)}(\bar{a}-\bar{x}) .
\end{aligned}
$$

Therefore, if $|\bar{a}-\bar{b}| \leq \frac{|\bar{x}-\bar{a}|}{2}$, then

$$
\left|f_{t} * \Gamma_{s}(x)\right| \leq C(s+t)^{-1 / 2}|\bar{b}-\bar{a}| \bar{\Gamma}_{4(s+t)}(\bar{a}-\bar{x}) e^{-\frac{a_{n}^{2}}{4 t}} \Gamma_{s+t}^{n}\left(x_{n}\right) .
$$

Choose $0<\alpha<1$. Recall that

$$
\bar{\Gamma}_{4(t+s)}(\bar{a}-\bar{x}) \leq C(s+t)^{(1-\alpha) / 2}|\bar{a}-\bar{x}|^{-n+\alpha},
$$

and

$$
\Gamma_{s+t}^{n}\left(x_{n}\right) \leq \min \left\{C(s+t)^{-\frac{1}{2}}, C(s+t)^{\frac{\alpha}{2}} x_{n}^{-1-\alpha}\right\} .
$$

Hence if $|\bar{a}-\bar{b}| \leq \frac{|\bar{x}-\bar{a}|}{2}$, then

$$
\left|f_{t} * \Gamma_{s}(x)\right| \leq C|\bar{b}-\bar{a}| e^{-\frac{a_{n}^{2}}{4 t}}\left\{\begin{array}{l}
(s+t)^{-\frac{1}{2}-\frac{\alpha}{2}}|\bar{a}-\bar{x}|^{-n+\alpha}, \\
|\bar{a}-\bar{x}|^{-n+\alpha} x_{n}^{-1-\alpha},
\end{array}\right.
$$

which implies that

$$
\mathcal{N} f_{t}(x) \leq C|\bar{b}-\bar{a}| e^{-\frac{a_{n}^{2}}{4 t}}\left\{\begin{array}{l}
t^{-\frac{1}{2}+\frac{\alpha}{2}}|\bar{a}-\bar{x}|^{-n+\alpha} \\
|\bar{a}-\bar{x}|^{-n+\alpha} x_{n}^{-1-\alpha}
\end{array}\right.
$$

We also note that

$$
\begin{aligned}
\left|f_{t} * \Gamma_{s}(x)\right| & \leq C\left|\bar{\Gamma}_{t+s}(\bar{a}-\bar{x})-\bar{\Gamma}_{t+s}(\bar{b}-\bar{x})\right| e^{-\frac{a_{n}^{2}}{4 t}} \Gamma_{s+t}^{n}\left(x_{n}\right) \\
& \leq C\left(\left|\bar{\Gamma}_{t+s}(\bar{a}-\bar{x})\right|+\left|\bar{\Gamma}_{t+s}(\bar{b}-\bar{x})\right|\right) e^{-\frac{a_{n}^{2}}{4 t}} \Gamma_{s+t}^{n}\left(x_{n}\right) \\
& \leq C\left[\bar{\Gamma}_{2(s+t)}(\bar{a}-\bar{x})+\bar{\Gamma}_{2(s+t)}(\bar{b}-\bar{x})\right] e^{-\frac{a_{n}^{2}}{4 t}} \Gamma_{s+t}^{n}\left(x_{n}\right) .
\end{aligned}
$$

Note that

$$
\bar{\Gamma}_{2(t+s)}(\bar{a}-\bar{x}) \leq C(s+t)^{-\frac{\alpha}{2}}|\bar{a}-\bar{x}|^{-n+1+\alpha}
$$

and

$$
\Gamma_{t+s}^{n}\left(x_{n}\right) \leq \min \left\{C(s+t)^{-\frac{1}{2}}, C(t+s)^{\frac{\alpha}{2}} x_{n}^{-1-\alpha}\right\} .
$$

Hence, we also have

$$
\left|f_{t} * \Gamma_{s}(x)\right| \leq C e^{-\frac{a_{n}^{2}}{4 t}}\left\{\begin{array}{l}
(s+t)^{-\frac{1}{2}-\frac{\alpha}{2}}|\bar{a}-\bar{x}|^{-n+1+\alpha} \\
|\bar{a}-\bar{x}|^{-n+1+\alpha} x_{n}^{-1-\alpha}
\end{array}\right.
$$


which implies that

$$
\mathcal{N} f_{t}(x) \leq C e^{-\frac{a_{n}^{2}}{4 t}}\left\{\begin{array}{l}
t^{-\frac{\alpha}{2}}|\bar{a}-\bar{x}|^{-n+1+\alpha} \\
|\bar{a}-\bar{x}|^{-n+1+\alpha} x_{n}^{-1-\alpha}
\end{array}\right.
$$

Now we estimate the $L^{1}$ norm of $\mathcal{N} f_{t}$. For $|\bar{a}-\bar{b}| \leq \frac{|\bar{x}-\bar{a}|}{2}$, we make use of the estimate (C.28) and for $|\bar{a}-\bar{b}| \geq \frac{|\bar{x}-\bar{a}|}{2}$ we make use of the estimate (C.29).

Decompose the domain $\mathbb{R}_{+}^{n}$ into four parts $D_{i}, i=1, \ldots, 4$, where

$$
\begin{aligned}
& D_{1}=\left\{\left(\bar{z}, x_{n}\right):|\bar{x}-\bar{a}| \geq 2|\bar{a}-\bar{b}|,\left|x_{n}\right| \geq \sqrt{t}\right\}, \\
& D_{2}=\left\{\left(\bar{z}, x_{n}\right):|\bar{x}-\bar{a}| \geq 2|\bar{a}-\bar{b}|,\left|x_{n}\right| \leq \sqrt{t}\right\}, \\
& D_{3}=\left\{\left(\bar{z}, x_{n}\right):|\bar{x}-\bar{a}| \leq 2|\bar{a}-\bar{b}|,\left|x_{n}\right| \geq \sqrt{t}\right\}, \\
& D_{4}=\left\{\left(\bar{z}, x_{n}\right):|\bar{x}-\bar{a}| \leq 2|\bar{a}-\bar{b}|,\left|x_{n}\right| \leq \sqrt{t}\right\} .
\end{aligned}
$$

Then we have that

$$
\begin{aligned}
& \int_{\mathbb{R}^{n}} \mathcal{N} f_{t}(x) d x= \sum_{i=1}^{4} \int_{D_{i}} \mathcal{N} f_{t}(x) d x \\
& \leq C e^{-\frac{a_{n}^{2}}{4 t}} {\left[\int_{D_{1}}|\bar{a}-\bar{b}||\bar{a}-\bar{x}|^{-n+\alpha} x_{n}^{-1-\alpha} d x\right.} \\
&+\int_{D_{2}}|\bar{a}-\bar{b}| t^{-\frac{1}{2}-\frac{\alpha}{2}}|\bar{a}-\bar{x}|^{-n+\alpha} d x \\
&+\int_{D_{3}}|\bar{a}-\bar{x}|^{-n+1+\alpha} x_{n}^{-1-\alpha} d x \\
&\left.+\int_{D_{4}} t^{-\frac{1}{2}-\frac{\alpha}{2}}|\bar{a}-\bar{x}|^{-n+1+\alpha} d x\right] \\
& \leq C e^{-\frac{a_{n}^{2}}{4 t}}|\bar{a}-\bar{b}|^{\alpha} t^{-\frac{\alpha}{2}}
\end{aligned}
$$

Therefore, we conclude that

$$
f_{t}=1_{\mathbb{R}_{+}^{n}}\left[\tau_{a^{*}} \Gamma_{t}-\tau_{b^{*}} \Gamma_{t}\right] \in \mathcal{H}^{1}\left(\mathbb{R}^{n}\right)
$$

\section{Appendix D. Proof of Lemma 2.7.}

Set

$$
\begin{aligned}
(a) & =\int_{0}^{x_{n} / 2} \int_{\mathbb{R}^{n-1}} \partial_{x_{i}} \partial_{x_{j}} N(x-z) f(z, y) d z \\
(b) & =\int_{x_{n} / 2}^{x_{n}} \int_{\mathbb{R}^{n-1}} \partial_{x_{i}} \partial_{x_{j}} N(x-z)\left[f(z, y)-f\left(\bar{x}, z_{n}, y\right)\right] d z, \quad \text { and } \\
(c) & =\int_{x_{n} / 2}^{x_{n}} f\left(\bar{x}, z_{n}, y\right) \int_{\mathbb{R}^{n-1}} \partial_{x_{i}} \partial_{x_{j}} N(x-z) d \bar{z} d z_{n} .
\end{aligned}
$$


Then

$$
\int_{0}^{x_{n}} \int_{\mathbb{R}^{n-1}} \partial_{x_{i}} \partial_{x_{j}} N(x-z) f(z, y) d z=(a)+(b)+(c) .
$$

Since $\left|\partial_{x_{i}} \partial_{x_{j}} N(x-z)\right| \leq C|x-z|^{-n}$ and $\int_{\mathbb{R}^{n-1}}|x-z|^{-n} d \bar{z}=\frac{C}{\left|x_{n}-z_{n}\right|}$, we have

$$
\begin{aligned}
\int_{\mathbb{R}_{+}^{n}}(a) d y & \leq C\left(\int_{0}^{x_{n} / 2} \frac{1}{x_{n}-z_{n}} d z_{n}\right)\left(\sup _{z \in \mathbb{R}_{+}^{n}} \int_{\mathbb{R}_{+}^{n}}|f(z, y)| d y\right) \\
& \leq C \sup _{z \in \mathbb{R}_{+}^{n}} \int_{\mathbb{R}_{+}^{n}}|f(z, y)| d y .
\end{aligned}
$$

Since, for $0<\alpha<1$,

$$
\begin{aligned}
& \left|\partial_{x_{i}} \partial_{x_{j}} N(x-z)\left[f(z, y)-f\left(\bar{x}, z_{n}, y\right)\right]\right| \\
\leq & C|x-z|^{\alpha}|x-z|^{-n} \frac{\left|f\left(\bar{z}, z_{n}, y\right)-f\left(\bar{x}, z_{n}, y\right)\right|}{|x-z|^{\alpha}} \\
\leq & C\left|x_{n}-z_{n}\right|^{\alpha}|x-z|^{-n} \frac{\left|f\left(\bar{z}, z_{n}, y\right)-f\left(\bar{x}, z_{n}, y\right)\right|}{|\bar{x}-\bar{z}|^{\alpha}}
\end{aligned}
$$

we obtain that

$$
\begin{aligned}
& \int_{\mathbb{R}_{+}^{n}}(b) d y \\
\leq & C \int_{\frac{x_{n}}{2}}^{x_{n}}\left(x_{n}-z_{n}\right)^{\alpha} \int_{\mathbb{R}^{n-1}}|x-z|^{-n}\left[\sup _{\bar{z} \in \mathbb{R}^{n-1}} \int_{\mathbb{R}_{+}^{n}} \frac{\left|f\left(\bar{z}, z_{n}, y\right)-f\left(\bar{x}, z_{n}, y\right)\right|}{|\bar{x}-\bar{z}|^{\alpha}} d y\right] d \bar{z} d z_{n} \\
\leq & C x_{n}^{\alpha+1}\left(\int_{\mathbb{R}^{n-1}}|x-z|^{-n} d \bar{z}\right)\left(\sup _{x_{n} / 2<z_{n}<x_{n}} \sup _{\bar{z} \in \mathbb{R}^{n-1}} \int_{\mathbb{R}_{+}^{n}} \frac{\left|f\left(\bar{z}, z_{n}, y\right)-f\left(\bar{x}, z_{n}, y\right)\right|}{|\bar{x}-\bar{z}|^{\alpha}} d y\right) \\
\leq & C x_{n}^{\alpha}\left(\sup _{x_{n} / 2<z_{n}<x_{n}} \sup _{\bar{z} \in \mathbb{R}^{n-1}} \int_{\mathbb{R}_{+}^{n}} \frac{\left|f\left(\bar{z}, z_{n}, y\right)-f\left(\bar{x}, z_{n}, y\right)\right|}{|\bar{x}-\bar{z}|^{\alpha}} d y\right) .
\end{aligned}
$$

Now we would like to show that $(c)=0$. Note that

$$
p . v . \int_{\mathbb{R}^{n-1}} \frac{\partial^{2}}{\partial y_{i} \partial y_{j}} N(x-y) d \bar{y}=\lim _{\epsilon \rightarrow 0, R \rightarrow \infty} \int_{\epsilon \leq|\bar{x}-\bar{y}| \leq R} \frac{\partial^{2}}{\partial y_{i} \partial y_{j}} N(x-y) d S_{\bar{y}}
$$

and

$$
\begin{aligned}
& \int_{\epsilon \leq|\bar{x}-\bar{y}| \leq R} \frac{\partial^{2}}{\partial y_{i} \partial y_{j}} N(x-y) d \bar{y} \\
= & \int_{S_{R}(\bar{x})} \partial_{y_{i}} N(x-y) n_{j} d S_{\bar{y}}-\int_{S_{\epsilon}(\bar{x})} \partial_{y_{i}} N(x-y) n_{j} d S_{\bar{y}} .
\end{aligned}
$$

Here $S_{R}(\bar{x})=\left\{\bar{y} \in \mathbb{R}^{n-1}:|\bar{x}-\bar{y}|=R\right\}$ and $n_{j}=\frac{y_{j}-x_{j}}{|\bar{y}-\bar{x}|}$ is the $j$-th component of the unit outer normal vector. If $i=1, \ldots, n-1$, then

$$
\left|\int_{S_{R}(\bar{x})} \partial_{y_{i}} N(x-y) n_{j} d S_{\bar{y}}\right|=\left|\int_{S_{R}(\bar{x})} \frac{y_{i}-x_{i}}{|x-y|^{n}} \frac{y_{j}-x_{j}}{|\bar{y}-\bar{x}|} d S_{\bar{y}}\right|
$$




$$
=\left|\int_{S_{n-2}} \frac{w_{i} w_{j}}{n \omega_{n}\left(R^{2}+\left(x_{n}-y_{n}\right)^{2}\right)^{n / 2}} R^{n-1} d S_{w}\right| \leq \frac{C}{R} \rightarrow 0 \quad \text { as } R \rightarrow \infty,
$$

where $S_{n-2}$ is the unit sphere in $\mathbb{R}^{n-1}, w_{i}=\frac{y_{i}}{|\bar{y}|}$ is the outward unit normal vector to $S_{n-2}$, and

$$
\begin{aligned}
& \left|\int_{S_{\epsilon}(\bar{x})} \partial_{y_{i}} N(x-y) n_{j} d S_{\bar{y}}\right| \\
= & \left|\int_{S_{n-2}} \frac{w_{i} w_{j}}{n \omega_{n}\left(\epsilon^{2}+\left(x_{n}-y_{n}\right)^{2}\right)^{n / 2}} \epsilon^{n-1} d S_{\bar{y}}\right| \leq C \frac{\epsilon^{n-1}}{\left|x_{n}-y_{n}\right|^{n}} \rightarrow 0 \quad \text { as } \epsilon \rightarrow 0 .
\end{aligned}
$$

If $i=n$, then

$$
\int_{S_{R}(\bar{x})} \partial_{y_{i}} N(x-y) n_{j} d S_{\bar{y}}=0
$$

since

$$
\begin{aligned}
\int_{S_{R}(\bar{x})} \partial_{y_{i}} N(x-y) n_{j} d S_{\bar{y}} & =\int_{S_{n-2}} \frac{\left(x_{n}-y_{n}\right) w_{j}}{n \omega_{n}\left(R^{2}+\left(x_{n}-y_{n}\right)^{2}\right)^{n / 2}} R^{n-2} d S_{\bar{y}} \\
& =\frac{\left(x_{n}-y_{n}\right) R^{n-2}}{n \omega_{n}\left(R^{2}+\left(x_{n}-y_{n}\right)^{2}\right)^{n / 2}} \int_{S_{n-2}} w_{j} d S_{\bar{y}}=0 .
\end{aligned}
$$

Likewise,

This implies that

$$
\int_{S_{\epsilon}(\bar{x})} \partial_{y_{i}} N(x-y) n_{j} d S_{\bar{y}}=0
$$

$$
\int_{\mathbb{R}^{n-1}} \partial_{y_{i}} \partial_{y_{j}} N(x-y) d \bar{y}=0,
$$

and this again implies that $(c)=0$.

\section{References}

[1] H. Amann, On the strong solvability of the Navier-Stokes equations, J. Math. Fluid Mech. 2 (2000), no. 1, 16-98.

[2] H.-O. Bae, Temporal and spatial decays for the Stokes flow, J. Math. Fluid Mech. 10 (2008), no. 4, 503-530.

[3] _ Temporal decays in $L^{1}$ and $L^{\infty}$ for the Stokes flow, J. Differential Equations 222 (2006), no. 1, 1-20.

[4] W. Borchers and T. Miyakawa, $L^{2}$ decay for the Navier-Stokes flow in halfspaces, Math. Ann. 282 (1988), no. 1, 139-155.

[5] F. Crispo and P. Maremonti, On the $(x, t)$ asymptotic properties of solutions of the Navier-Stokes equations in the half space, Zap. Nauchn. Sem. POMI, 318 (2004); translation in J. Math. Sci. 136 (2006), no. 2, 3735-3767.

[6] Y. Fujigaki and T. Miyakawa, Asymptotic profiles of nonstationary incompressible Navier-Stokes flows in the half-space, Methods Appl. Anal. 8 (2001), no. 1, 121-157.

[7] Y. Giga, S. Matsui, and O. Sawada, Global existence of two-dimensional Navier-Stokes flow with nondecaying initial velocity, J. Math. Fluid Mech. 3 (2001), no. 3, 302-315.

[8] Y. Giga, S. Matsui, and Y. Shimizu, On estimates in Hardy spaces for the Stokes flow in a half-space, Math. Z. 231 (1999), no. 2, 383-396. 
[9] Y. Giga, K. Inui, A. Mahalov, A. Matsui, and J. Saal, Rotating Navier-Stokes equations in $\mathbb{R}_{+}^{3}$ with initial data nondecreasing at infinity: the Ekman boundary layer problem, Arch. Ration. Mech. Anal. 186 (2007), no. 2, 177-224.

[10] M. Hieber and O. Sawada, The Navier-Stokes equations in $\mathbb{R}^{n}$ with linearly growing initial data, Arch. Ration. Mech. Anal. 175 (2005), no. 2, 269-285.

[11] J. Kato, The uniqueness of nondecaying solutions for the Navier-Stokes equations, Arch. Ration. Mech. Anal. 169 (2003), no. 2, 159-175.

[12] P. Maremonti, Stokes and Navier-Stokes problems in a half space: the existence and uniqueness of solutions a priori nonconvergent to a limit at infinity, Journal of Mathematical Sciences 159 (2009), no. 4, 486-523.

[13] P. Maremonti and G. Starita, Nonstationary Stokes equations in a half-space with continuous initial data, Zap. Nauchn. Sem. POMI, 295; translation in J. Math. Sci. 127 (2005), no. 2, 1886-1914.

[14] E. Nakai and T. Yoneda, Generalized Campanato spaces and the uniqueness of nondecaying solutions for the Navier-Stokes equations, preprint, 2009.

[15] Y. Shimizu, L $\infty$ estimate of the first-order space derivatives of Stokes flow in a half space, Funkcial. Ekvac. 42 (1999), no. 2, 291-309.

[16] V. A. Solonnikov, On nonstationary Stokes problem and Navier-Stokes problem in a halfspace with initial data nondecreasing at infinity, Problemy Matematisheskogo Analiza, (2003) no. 25, 189-210; translation in J. Math. Sci. (N. Y.) 114 (2003), no. 5, 1726-1740.

[17] S. Ukai, A solution formula for the Stokes equation in $\mathbf{R}_{+}^{n}$, Comm. Pure Appl. Math. 40 (1987), no. 5, 611-621.

HyeONG-OHK BAE

Department of Financial EngineERing

AJOU UNIVERSITY

Suwon 443-749, Korea

E-mail address: hobae@ajou.ac.kr

Bum JA Jin

Department of Mathematics

MokPo National University

Muan 534-729, Korea

E-mail address: bumjajin@hanmail.net 